\title{
Optimal Estimation of Shear Strength Parameters Based on Copula Theory Coupling Information Diffusion Technique
}

\author{
Xinlong Zhou, ${ }^{1,2}$ Guang Zhang, ${ }^{1,2}$ Shaohua $\mathrm{Hu} \mathbb{D}^{1,2,3}$ and Junzhe Li ${ }^{1,2}$ \\ ${ }^{1}$ School of Safety Science and Emergency Management, Wuhan University of Technology, 122 Luoshi Road, Wuhan, \\ Hubei 430070, China \\ ${ }^{2}$ School of Resources and Environmental Engineering, Wuhan University of Technology, 122 Luoshi Road, Wuhan, \\ Hubei 430070, China \\ ${ }^{3}$ State Key Laboratory of Safety and Health for Metal Mines, Maanshan 243000, China
}

Correspondence should be addressed to Shaohua Hu; sh_kxin@whu.edu.cn

Received 24 November 2018; Revised 31 March 2019; Accepted 16 April 2019; Published 26 May 2019

Academic Editor: Jian Ji

Copyright $\odot 2019$ Xinlong Zhou et al. This is an open access article distributed under the Creative Commons Attribution License, which permits unrestricted use, distribution, and reproduction in any medium, provided the original work is properly cited.

In geotechnical reliability analysis, random volatility in marginal distributions of shear strength parameters has been rarely considered. Unfortunately, conventional marginal distribution models cannot characterize real probability distribution accurately, leading to considerable dispersion with incomplete probabilistic information. In this paper, an estimation methodology is proposed based on copula theory coupling information diffusion technique. Firstly, information diffusion distribution is extended to represent one-dimensional marginal distributions of shear strength parameters. Secondly, copula theory is employed to characterize the dependence structures among the parameters. Eventually, equivalent sample is yielded by information diffusion distribution that has been already established. A case study in Singapore is implemented to enunciate and validate the competence of the proposed method. The performances of the candidate copulas coupling different marginal distributions are further discussed. Results indicate that information diffusion distribution can efficiently capture the random volatility of real distributions of shear strength parameters and hold remarkable superiority in modeling marginal distributions. The equivalent sample, estimated by information diffusion technique in conjunction with Gaussian copula, has considerable consistency with original data. The proposed method can provide a reference to reliability analysis in geotechnical engineering.

\section{Introduction}

It is well recognized that shear strength parameters (cohesive force $c$ and internal friction angle $\varphi$ ) are significantly crucial to geotechnical reliability analysis [1-4]. In geotechnical engineering, $c$ and $\varphi$ are often viewed as random variables, and their joint cumulative distribution function (CDF) or probability density function (PDF) seriously affects the accuracy of risk assessment $[5,6]$. In order to conduct a realistic analysis on the geotechnical reliability, it is essential to accurately address the joint CDF or PDF of the shear strength parameters. However, only limited data can be obtained by the field test or laboratory test. Under these incomplete information, marginal distributions and correlation coefficients are approximately estimated with inevitable uncertainty $[7,8]$. The joint $\mathrm{CDF}$ or PDF is also challenged by data scarcity and uncertainty, leading to a large dispersion in the probability of failure.

Traditionally, bivariate normal distribution [9], bivariate Beta distribution [10], and Nataf distribution [11] are commonly employed to address that objective. Nevertheless, such approaches are available under the constraint that all the variables correspond to normal distribution or Beta distribution. And, Nataf distribution inherently assumes a Gaussian dependence structure for the random variables, which can be inappropriate in some cases [8]. Recently, the copula approach, considering the deduction of marginal distribution and the selection of optimal copula function separately, provides a fairly general way for modeling joint distribution [12-14]. A copula is a function that maps the joint distribution of variables with their one-dimensional marginal distributions $[15,16]$. Arbitrary marginal distribution and 
corresponding dependence structure can be incorporated by it. In recent years, it has been progressively applied to reliability analysis in geotechnical engineering. Zhang et al. performed slope reliability by counting copula-based bivariate distribution of shear strength parameters [7]. Wu employed the Gaussian and Frank copulas to construct the joint distribution among cohesion, friction angle, and unit weight of soils [12]. Motamedi and Liang conducted a landslide hazard assessment using the Copula modeling technique [13]. Das et al. introduced the copula theory to study the reliability of vegetated slopes [17].

It is worthwhile noting that the copula is not a panacea, though its connotation has been enriched and developed along with above researchers' efforts. In Sklar's theorem [18], copulas generate random pairs in rank space, and then, equivalent samples can be simulated by corresponding marginal distributions [8]. Therefore, the selection of marginal distribution types for shear strength parameters has a direct effect on the calculated slope reliability. Chen et al. had proven that marginal distribution has a minor effect on calculated results of the factor of safety as a major influence on the failure probability [19]. Wu demonstrated the performances of conventional distribution models differ from each other [20]. These studies concluded that a reasonable marginal distribution of shear strength parameter is vitally significant to the accuracy of reliability analysis. Presently, the deduction of marginal distribution for individual parameter remains subjective and open to debate. Various statistical models have been proposed and utilized for fitting the base distribution of shear strength parameters. For instance, Low [21], Li et al. [22], Ji et al. [23], and Zhou et al. [24] employed normal distribution to represent base distribution of shear strength parameters. Brejda et al. [25], Fenton and Griffiths [26], and Jiang et al. [27] considered the parameters obeying lognormal distribution because the soil properties were strictly nonnegative. Harrop-Williams [28] and Harr [29] demonstrated that beta distribution was another suitable choice for distribution properties. Other distributions, such as Gamma [12, 30], Gumbel [31, 32], and Weibull [20,33], are gaining popularity. These assumptions, however, do not always hold as the random volatility owing to the heterogeneity of rock or soil mass is observed in real distribution $[4,34,35]$. In modeling the base distributions realistically, it may be necessary to recreate the random volatility by fitting a sufficiently flexible theoretical probability distribution to shear strength parameters. Furthermore, the deduction of marginal distribution is commonly subject to sparse sample, and reliability analysis thus has associated uncertainties, leading to a high deviation from reality. However, these specific characters in marginal distribution estimation have been rarely accounted for. The aforementioned conventional mathematical approaches cannot truly cater to random volatility nature because their varying curves act as a single peak value wave. Once the selected model does not coincide with the real distribution, it cannot asymptotically represent the actual properties as the sample size and computational capability increase. Accordingly, it is imperative to explore some novel deduction method for geotechnical parameters under incomplete information.
Information diffusion (ID) technique is inherently a setvalued fuzzy mathematical processing method [36]. It maintains that each information sample point is inclined to develop into multiple information points in the process of transition from incompleteness to completeness. In this respect, single-valued samples can be expanded to set-valued samples through a certain diffusion function. Consequently, the corresponding information expansion of incomplete systems can be achieved. It is perfectly capable of incomplete information processing and avoids solving the membership function. Based on the information diffusion theory, Gong et al. [37] and Huang et al. [38] specified information diffusion distribution and successfully captured the random volatility of geotechnical parameters, providing a new enlightenment to marginal distribution deduction. However, these studies did not perform model construction for multivariate distribution. The application and effectiveness of ID approach coupling copula theory under incomplete probabilistic information remain to be validated in a rigorous way.

In this case, a novel estimation method for shear strength parameters is proposed. The information diffusion technique is further explored to deduce the optimal marginal distributions of shear strength parameters, in conjunction with copula theory employed to model the dependence structure among them. For this objective, the rest of the study is organized as follows. First, copula theory and construction procedure of joint distribution are briefly elucidated in Section 2. Then, information diffusion distribution of each shear strength parameters is constructed and validated by mathematical and graphic analysis in Section 3. The whole implementation procedure is induced in Section 4. Sequentially, Section 5 gives an illustrative example to demonstrate the performance of the proposed method. To validate the consistency between equivalent and initial samples, a backward analysis is conducted in this section. Finally, Section 6 provides the conclusions and suggestions for future work.

\section{Joint Distribution of Shear Strength Parameters Based on Copulas}

2.1. Bivariate Distribution of $c$ and $\varphi$ Using Copula Theory. In Sklar's theorem [18], a bivariate $\operatorname{CDF}, F(c, \varphi)$, can be expressed in terms of a copula function $C\left(u_{1}, u_{2} ; \theta\right)$ and two marginal distributions $u_{1}=F_{1}(c)$ and $u_{2}=F_{2}(\varphi)$ :

$$
F(c, \varphi)=C\left(F_{1}(c), F_{2}(\varphi) ; \theta\right)=C\left(u_{1}, u_{2} ; \theta\right),
$$

where $\theta$ is the related parameter of copula function $C(\cdot)$. If $u_{1}$ and $u_{2}$ are continuous, $C(\cdot)$ can be uniquely determined.

By taking derivatives of equation (1), the bivariate joint PDF $f(c, \varphi)$ can be given in terms of a copula probability density function $c(\cdot)$ in the following form:

$$
f(c, \varphi)=f_{1}(c) f_{2}(\varphi) c\left(F_{1}(c), F_{2}(\varphi) ; \theta\right) .
$$

By definition, the construction of the copula joint distribution function can be broadly converted into two steps: First, the probable marginal distributions of $c$ and $\varphi$ are determined. As mentioned in Introduction, this work is 
basic and vitally significant to the rest of reliability study. Detailed analysis is given in the next section. Second, the optimal copula function to characterize the dependence structure in the original data is identified. Previous studies have established the fact that different copula functions characterize different dependence structures, which is quantified by the correlation coefficient [39]. Therein, Pearson linear correlation coefficient $\gamma_{n}$ and Kendall's rank correlation coefficient $\tau_{n}$ are widely derived.

Assume that $\left(x_{i}, y_{i}\right)(i=1,2, \ldots, n)$ are $n$-size observation samples from population $(X, Y) . \gamma_{n}$ and $\tau_{n}$ can be, respectively, expressed as follows [18]:

$$
\begin{aligned}
\gamma_{n} & =\frac{\sum_{i=1}^{n}\left(x_{i}-\bar{x}\right)\left(y_{i}-\bar{y}\right)}{(n-1) \sqrt{S_{x}^{2} S_{y}^{2}}}, \\
\tau_{n} & =\left(\begin{array}{c}
n \\
2
\end{array}\right)^{-1} \sum_{i<j} \operatorname{sgn}\left[\left(x_{i}-x_{j}\right)\left(y_{i}-y_{j}\right)\right],
\end{aligned}
$$

wherein $n$ is the number of observation samples $(X, Y), \bar{x}$ and $\bar{y}$ are the sample means, $S_{x}^{2}$ and $S_{y}^{2}$ are the sample variances, $\operatorname{sgn}(\cdot)$ is the sign function, $\operatorname{sgn}(\cdot)=1$ if $\left(x_{i}-x_{j}\right)\left(y_{i}-y_{j}\right)>0$, and $\operatorname{sgn}(\cdot)=-1$ if $\left(x_{i}-x_{j}\right)\left(y_{i}-y_{j}\right)<0, i, j=1,2, \ldots, n$. With respect to sample pairs $\left(x_{i}, y_{i}\right)$ and $\left(x_{j}, y_{j}\right)$, if they satisfy $\left(x_{i}-x_{j}\right)\left(y_{i}-y_{j}\right)$ $>0$, they are considered to be in accordance with each other.

To address the interrelationship between the two parameters, the observed data $(x, y)$ in original space are commonly transformed into the standard uniform distribution $u_{i}=\left(u_{1 i}, u_{2 i}\right)$, which can be computed by using the following equation [40]:

$$
\left\{\begin{array}{l}
u_{1 i}=\frac{\operatorname{rank}\left(x_{i}\right)}{n+1}, \\
u_{2 i}=\frac{\operatorname{rank}\left(y_{i}\right)}{n+1}, \quad i=1,2, \ldots, n,
\end{array}\right.
$$

where $\operatorname{rank}\left(x_{i}\right)$ and $\operatorname{rank}\left(y_{i}\right)$ are the ascending order of $x_{i}$ and $y_{i}$, respectively.

2.2. Estimation of Related Parameters of Copula Function. The estimation of related parameter $\theta$ is a key step in constructing copula function. It can be obtained according to the corresponding relationship between Kendall's rank correlation coefficient $\tau_{K}$ and copula function $C(\cdot)$, as shown in the following [18]:

$$
\tau_{K}=4 \int_{0}^{1} \int_{0}^{1} C\left(u_{1}, u_{2} ; \theta\right) d C\left(u_{1}, u_{2} ; \theta\right)-1 .
$$

Particularly, for Gaussian Copula functions, there is a simpler relationship:

$$
\tau_{K}=\frac{2 \arcsin (\rho)}{\pi},
$$

wherein $\rho$ is defined as the related parameter of Gaussian copula. By solving equation (5) or (6), each $\rho$ or $\theta$ of copula selected to fit the dependence structure between $c$ and $\varphi$ is obtained. Furthermore, unique copula function can be determined. Obviously, it can be recognized from equations (5) and (6) that the copula parameter is independent of their base distribution of $c$ and $\varphi$.

2.3. Identification of Optimal Copula Function. Squared Euclidean distance (SED) and Akaike information criterion (AIC) are routinely employed to sieve the optimal copula function. SED is defined as the quadratic sum of the $D$-value between the theoretical joint cumulative frequency $p$ and empirical joint cumulative frequency $p_{e}$, located in $F(x)$ and $F^{\prime}(x)$, respectively, denoted as $d_{2}$ in the following equation [41]:

$$
d_{2}=\sum_{i=1}^{n}\left(p_{i}-p_{e_{i}}\right)^{2}
$$

AIC is commonly used for the selection of optimal statistical model. Briefly, it can be expressed as [42]

$$
\mathrm{AIC}=2 k-2 \ln (L)
$$

where $k$ is the number of parameters of statistical model and $L$ is the maximized value of the likelihood function for the estimated model. Given a set of candidate models for the data, the preferred model is the one with the minimum AIC value. Substituting theoretical joint $\operatorname{CDF} F(x)$ and empirical joint CDF $F^{\prime}(x)$ into equation (8), the AIC of copula is given as

$$
\mathrm{AIC}=n \ln \left\{\frac{1}{n-k} \sum_{i=1}^{n}\left(F(x)-F^{\prime}(x)\right)^{2}\right\}+2 k .
$$

Generally, the copula function associated with minimum SED and AIC values is considered to be optimal.

\section{Probability Distribution Estimation Using Information Diffusion Technique}

As discussed earlier, marginal distribution of each parameter is significant to copula joint distribution construction. However, most studies estimate marginal distributions of shear strength parameters without considering random volatility owing to the heterogeneity of rock or soil mass. Moreover, incomplete probabilistic information severely restricts the performance of conventional models. Therefore, conventional marginal distribution models cannot characterize real probability distribution accurately, leading to considerable dispersion with incomplete probabilistic information. For this case, information diffusion technique is introduced to address a more appropriate distribution.

3.1. Information Diffusion Deduction Method. Let $X=\left(x_{1}, x_{2}, \ldots, x_{n}\right)$ be a sample set made up of $n$ elements and $U=\left(u_{1}, u_{2}, \ldots, u_{m}\right)$ be the domain of discourse made up of $m$ elements. Then, the nondiffusion estimate $\widehat{R}$ can be defined as follows [36, 43]:

$$
\widehat{R}(\gamma, X)=\left\{\gamma\left(\chi\left(x_{i}, u\right)\right) \mid x_{i} \in X, u \in U\right\},
$$

where $\gamma$ is called as reasonable operator and $\chi\left(x_{i}, u\right)$ is the associate characteristic function. 
If and only if $X$ is incomplete, there must exist a diffusion function $\mu\left(x_{i}, u\right)$ and a corresponding operator $\gamma^{\prime}$. Sequentially, the diffusion estimate can be expressed as

$$
\widetilde{R}\left[\gamma^{\prime}, D(X)\right]=\left\{\gamma^{\prime}\left(\mu\left(x_{i}, u\right)\right) \mid x_{i} \in X, u \in U\right\},
$$

that satisfies

$$
\|R-\widetilde{R}\|<\|R-\widehat{R}\|,
$$

where $\|\cdot\|$ is the absolute value of deviation between the estimated relationship and the real relationship.

In the above equations, ID technique ensures a specific diffusion function to improve nonspread estimate under incomplete information. Alternatively, when $X$ is incomplete, there must exist a diffusion function $\mu(\cdot)$ to extract and propagate fuzzy information of $X$ in order to more accurately estimate the function approximation of a relation $R$.

Figure 1 gives the explanation of the information diffusion principle.

Suppose that $\mu(\cdot)$ is a Borel measurable function in $(-\infty$, $+\infty)$.

Here, a normal diffusion function, as shown in equation (13), is adopted to diffuse the information retained by observation $x_{i}$ to the monitoring point $u_{j}$ in normal approach:

$$
\mu(x)=\frac{1}{h \sqrt{2 \pi}} \exp \left(-\frac{\left(x-x_{i}\right)^{2}}{2 h^{2}}\right),
$$

where $h$ is called as diffusion coefficient, determined by the maximum value, minimum value, and size of sample set. Note that $h$ affects the effectiveness of ID technique considerably. Some scholars have conducted a great amount of research to elucidate it. Particularly, Wang has given an extensive and mathematical investigation, as expressed in the following [44]:

$$
h=\frac{\gamma(b-a)}{n-1}
$$

where $a=\min \left\{x_{i}\right\}, b=\max \left\{x_{i}\right\}, n$ is the sample size, and $\zeta$ can be determined depending on the study of Wang [44], and the values for different sample size of $n$ are tabulated in Table 1.

Suppose $\mu(\cdot)$ is a Borel measurable function in $(-\infty$, $+\infty)$. In this case, one-dimension PDF is defined as follows:

$$
f\left(u_{j}\right)=\frac{1}{h \sqrt{2 \pi}} \sum_{i=1}^{n}\left\{\exp \left[-\frac{\left(u_{j}-x_{i}\right)^{2}}{2 h^{2}}\right]\right\} .
$$

3.2. Goodness-of-Fit Test. In order to examine the adequacy of ID approach, the goodness-of-fit test is absolutely essential. Herein, K-S test and AIC criteria are implemented for verification.

Assuming that $F_{0}(x)$ represents an estimated CDF, $F_{n}(x)$ is defined as the empirical CDF for $n$ observation samples. Let $D_{n}$ be the maximum $D$-value between $F_{0}(x)$ and $F_{n}(x)$, expressed as follows [45]:

$$
D_{n}=\max \left|F_{n}(x)-F_{0}(x)\right|,
$$

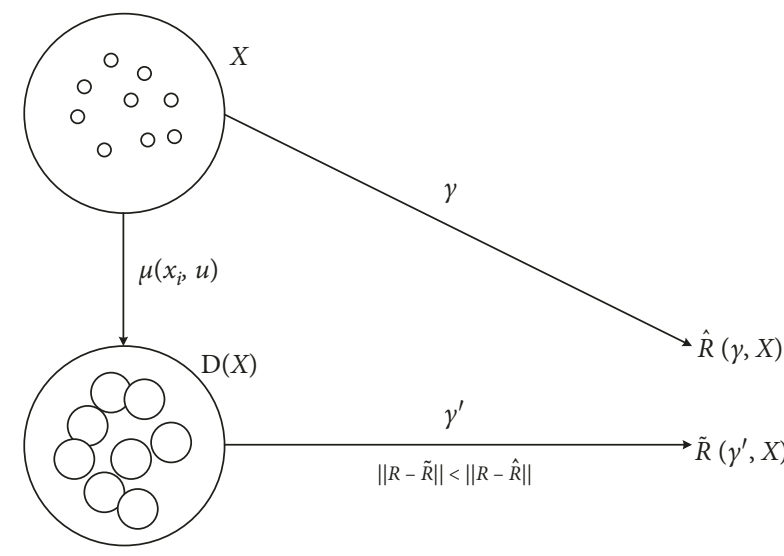

FIGURE 1: Schematic plot of information diffusion principle [43].

TABle 1: Values of $\zeta$ for different sample sizes of $n$.

\begin{tabular}{lc}
\hline$n$ & $\zeta$ \\
\hline 3 & 0.849321800 \\
4 & 1.273982782 \\
5 & 1.698643675 \\
6 & 1.336252561 \\
7 & 1.445461208 \\
8 & 1.395189816 \\
9 & 1.422962345 \\
10 & 1.416278786 \\
11 & 1.420835443 \\
12 & 1.420269570 \\
13 & 1.420698795 \\
14 & 1.420669671 \\
15 & 1.420693321 \\
16 & 1.420692226 \\
17 & 1.420693101 \\
17 & 1.420693101 \\
\hline
\end{tabular}

where $D_{n, \alpha}$ is called as the critical value of the Kolmogorov distribution. While $D_{n}<D_{n, \alpha}$, the estimated model population is considered to coincide with the estimated distribution, and vice versa.

AIC algorithm can be referred to Section 2.3. According to equation (9), each candidate case is encountered an AIC value. Specially, the candidate model with minimum AIC value is considered as the most probable marginal distribution.

\section{Implementation Procedure}

Based on the above analysis, the procedure of optimal estimation of shear strength parameters using copula theory coupling information diffusion technique can be divided into 5 steps as follows:

Step 1. Site-specific data of $c$ and $\varphi$ are obtained from the field or laboratory test.

Step 2. The optimal marginal distribution function is deduced. The coefficient $\zeta$ from Table 1 and compute diffusion 
coefficient $h$ are determined firstly. Then, by using equations (13)-(15), marginal PDFs and CDFs of single random variables $c$ and $\varphi$ are constructed with site-specific sample. Good-of-fit test is implemented for validation. For comparison, some conventional distributions can be derived simultaneously.

Step 3. The dependence structure between random variables is measured. Correlation coefficients between $c$ and $\varphi$ are calculated. And the most probable copula function with minimum AIC and SED is selected.

Step 4. Random pairs in rank space by the optimal copula function are simulated, which then yields equivalent samples via the prescribed marginal distribution.

Step 5. Backward analysis is performed for equivalent samples to verify the robustness and sensitivity of the proposed model.

Figure 2 portrays all crucial ingredients of the proposed model in a seamless manner.

\section{Illustrative Example}

5.1. Data Source and Property Analysis. Li et al. carried out a large number of uniaxial and a series of triaxial compression tests under different confining pressures [46]. The granite samples are all taken from Bukit Timah, Singapore. The specimen was made of $55 \mathrm{~mm}$ core, and the sample size was $30 \mathrm{~mm} \times 60 \mathrm{~mm}$. All tests were carried out on the RDT10000 dynamic load test apparatus. On the basis of these test data, Gong et al. constructed small sample sets of shear strength parameters according to the permutation and combination theory and Mohr-Coulomb shear strength criterion [37]. Next, a total of 22 pairs data were obtained using the robust regression estimation method. The correlation coefficients show that $\gamma_{n}=-0.8767$ and $\tau_{n}=-0.7843$. Sample means are $\mu_{c}=56.2939$ and $\mu_{\varphi}=41.7864$, respectively, and the corresponding standard deviations are $\sigma_{c}=9.4192$ and $\sigma_{\varphi}=2.0214$. Figure 3(a) shows the scatter plots of the observed data in original space and the uniformed data transformed by equation (4). It is explicit from not only Figure 3 but also coefficients $\gamma_{n}$ and $\tau_{n}$ that the original shear strength parameters $c$ and $\varphi$ are basically symmetry and there is a negative correlation between them. Thus, the proper joint distribution model studied in this paper should be capable of capturing the symmetry and negative correlation of the dependence structure between $c$ and $\varphi$.

For preliminary analysis, different marginal probability distributions, viz, normal distribution, lognormal distribution, extreme value distribution, and Weibull distribution, are examined on Matlab platform. Figure 4 gives their probability plots for the observed data. It can be readily derived that all the four conventional distributions cannot accurately model the probability distributions of shear strength parameters. As for $c$, the scatters loosely distribute and basically lie under the four fitting lines. More specifically, the scatters exhibit concentrated distribution at the middle as discrete dispersion at both tails. With respect to $\varphi$, scatters fluctuate up and down along the fitting lines. Likewise, they also concentrate locally and disperse at both tails of fitting lines, especially in Figures 4(c) and 4(d). Therefore, although the base distributions of shear strength parameters can be deduced by conventional distribution models, in many cases they cannot give unbiased and realistic probability estimations, and only the optimal probability distribution in a local range can be obtained. This is mainly because a sample size of $n=22$ is insufficient to perform an explicit description of individual distribution by them. Therefore, exploring a novel deduction approach to extract original information as much as possible is crucial. Fortunately, the information diffusion technique is capable of capturing and diffusing information original data retain, providing a new idea to address this problem.

\subsection{Optimal Marginal Distribution Deduction.}

Information diffusion technique is implemented to estimate PDFs and CDFs of $c$ and $\varphi$ from equation (15). Diffusion coefficient $h$ is determined in Table 2. The corresponding correlation coefficients are shown in Table 2 . The probability plots of $c$ and $\varphi$ are depicted in Figure 5. As a reference, the probability plots associated with normal distribution are also given in the axes. It can be clearly observed that the fitting curves associated with information diffusion distribution vary along with the scatter distribution of $c$ and $\varphi$. Almost all scatters distribute more smoothly along the fitting curves than those of normal distribution, indicating that the proposed approach can efficiently capture the variation characteristics of the real distributions.

To further verify the performance of the proposed method, four conventional distribution types are introduced as candidate models, i.e., truncated normal distribution (left truncated at zero), lognormal distribution, Gumbel distribution (left truncated at zero), and Weibull distribution. The aforementioned marginal distributions, along with the relationships between $(p, q)$ and $(\mu, \sigma)$, are summarized in Table 3 . Subsequently, AIC criteria and K-S test are employed to judge the adequacies of the candidate models, from equations (8) and (16), respectively. Here, due to the same sample size of $n=22$, the critical value is $D_{n, \alpha}=0.2809$, at the significance level of 0.05 . The results of good-of-fit test are given in Table 4. The values in bold denote minimum $D_{n}$ and AIC values.

It is evident that five values of $D_{n}$ obtained by corresponding marginal distribution functions are less than 0.2809. Namely, all five assumed models are statistically accepted at the significance level of 0.05 . This is attributed to the obstacle of fitting test method in practice. However, the magnitudes, representing corresponding fitting accuracies, vary from each other. As for $c$, the values of $D_{n}$ yielded by conventional margins belong to $(0.13,0.28)$, as that produced by ID approach dips to 0.0975 . There are $90.25 \%$ chances for original samples obeying ID distribution, implying that ID technique is much better than the other margins. Similarly, in terms of $\varphi$, ID distribution also has the least value of $D_{n}$, deeply reduced compared to the other cases. Obviously, not only $c$ but also $\varphi$, the PDF derived by ID approach can be complaint with actual distribution of 


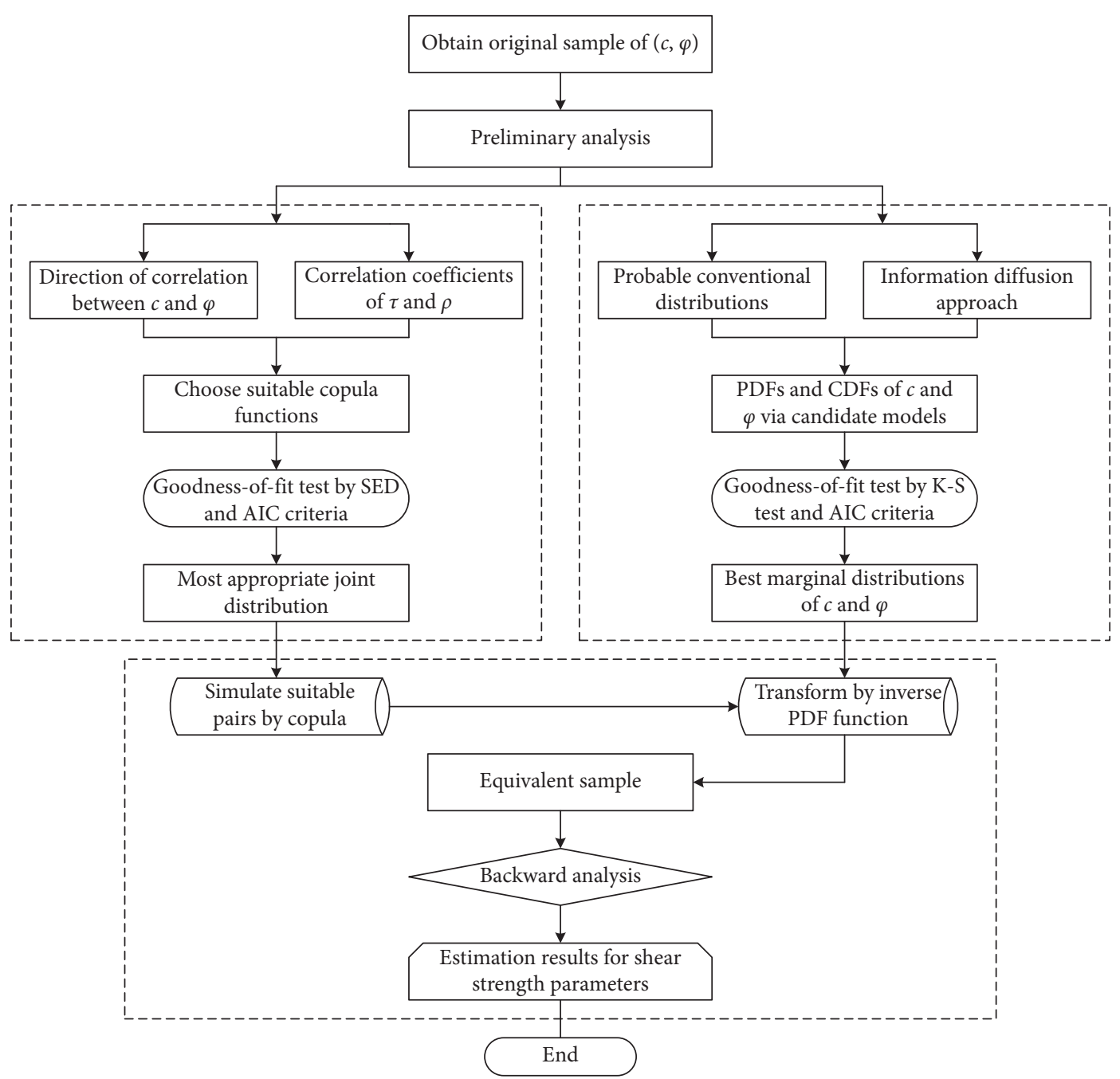

FIGURE 2: Flow diagram of optimal estimation model based on copula theory coupling information diffusion technique.

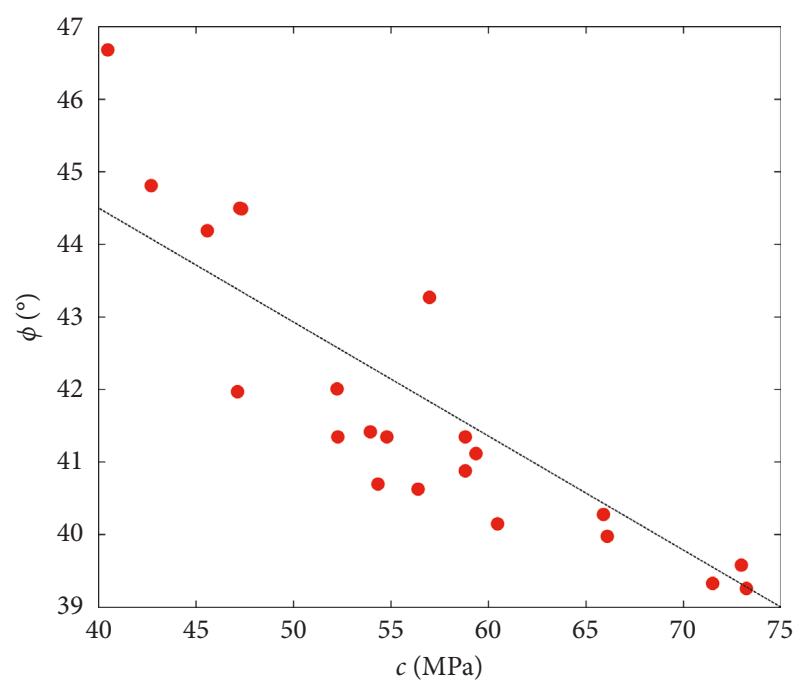

(a)

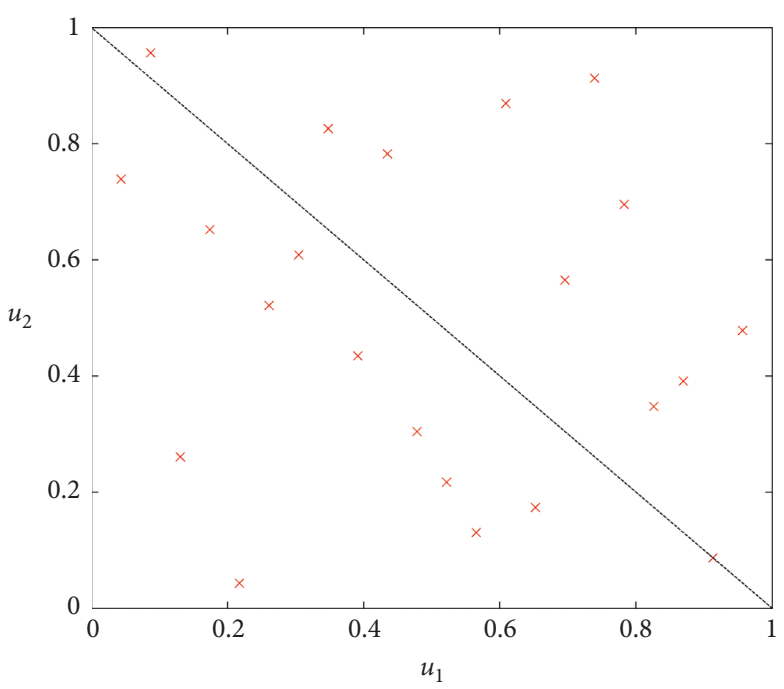

(b)

FiguRE 3: Scatter plots of (a) original data and (b) uniformed data. 


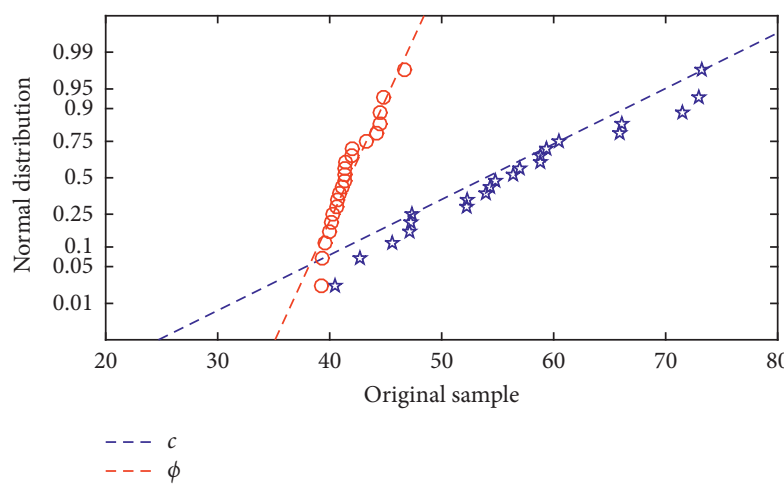

(a)

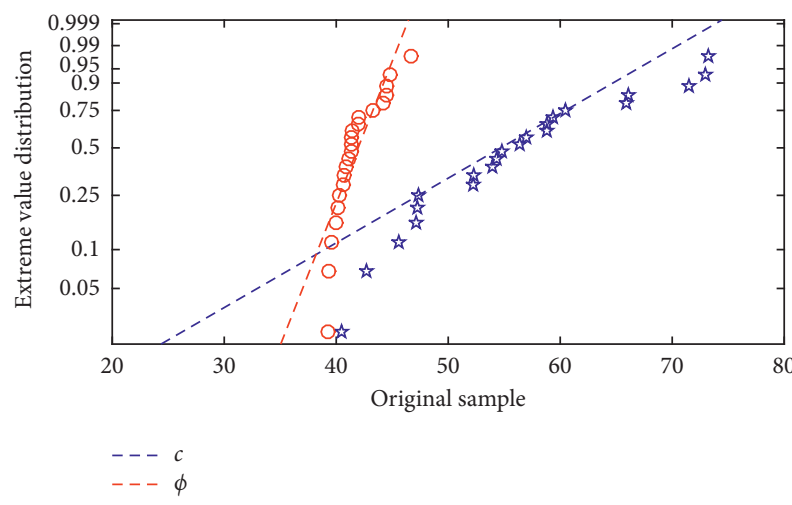

(c)

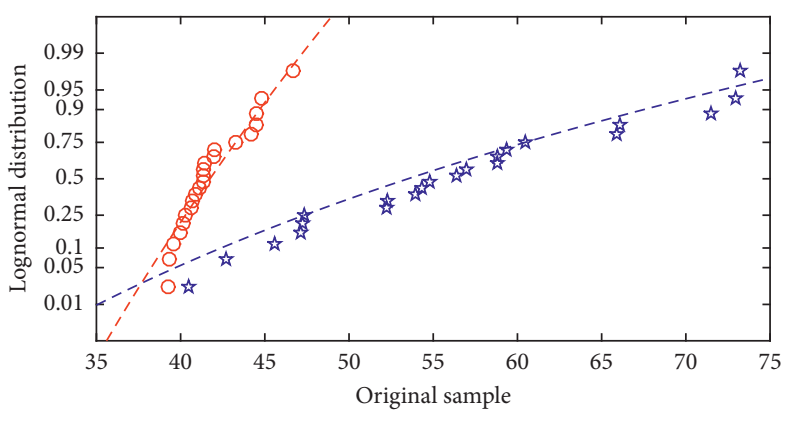

$--c$

-- $\phi$

(b)

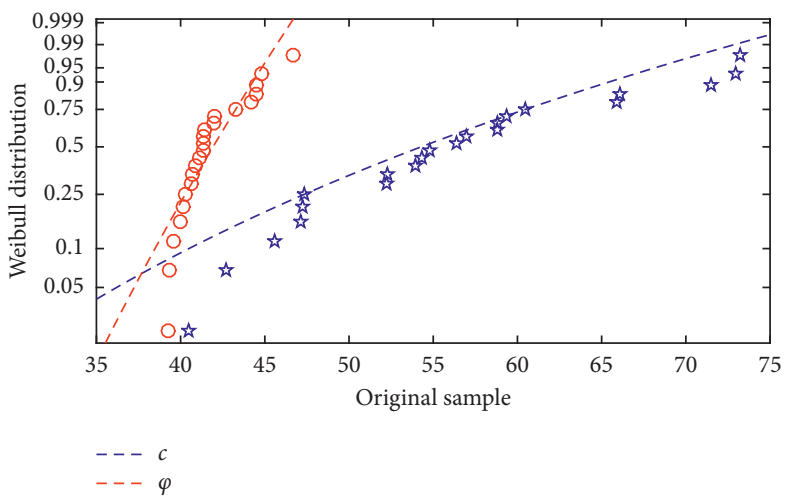

(d)

Figure 4: Probability plots of $c$ and $\varphi$ with different distribution types for (a) normal distribution, (b) lognormal distribution, (c) extreme value distribution, and (d) Weibull distribution.

TABle 2: Coefficients and PDFs of information diffusion distributions for $c$ and $\varphi$.

\begin{tabular}{lcccc}
\hline Parameter & $\Phi$ & $H$ & $\begin{array}{c}1 / \\
(2 \pi n h)\end{array}$ & $1 /\left(2 h^{2}\right)$ \\
\hline$C$ & 1.420693101 & 2.2154 & 0.0033 & 0.1019 \\
$\Phi$ & 1.420693101 & 0.5020 & 0.0144 & 1.9841 \\
\hline
\end{tabular}

random variables. In addition, among all of the available distributions, ID distribution has the minimum AIC value, as shown in Table 4. Both results indicate its best fit to actual observations.

Similar results can be drawn from graphic analysis. For visualization, the PDFs and CDFs of five candidate cases are depicted in Figures 6 and 7, respectively. In Figures 6(a) and $7(\mathrm{a})$, it can be readily derived that the histogram of $c$ and $\varphi$ exhibit evident volatility. This behavior enunciates the randomness and variability of shear strength parameters in realistic state. However, the PDFs associated with different candidate cases differ considerably. Particularly, each curve of classical ones merely has a single peak, which represents the maximum probability. Along the curve, probability point increases before the peak as decreases after that. Namely, they cannot describe the random volatility of initial data. As a contrary, the PDF curve depicted by ID approach holds multiple peaks, accurately capturing the variation trend and volatility of histogram. This is plainly due to the competences of extracting and diffusing original information of the novel approach. Moreover, it is worthwhile noting that the CDFs of information diffusion approximation are the best asymptote to the empirical CDFs of $c$ and $\varphi$, as shown in Figures 6(b) and 7(b).

Consequently, both mathematical and graphic analyses enunciate that ID distribution is the most probable marginal distribution for $c$ and $\varphi$, with a strong adequacy of capturing random volatility. A great improvement in deducing base distribution is achieved as compared to the conventional ones.

5.3. Bivariate Copula Distribution of $c$ and $\varphi$. Figure 3 and correlation coefficients have illuminated that $c$ and $\varphi$ exhibit negative correlation, indicating the direction of the dependence structure in the original data. As mentioned earlier, different correlation coefficient represents different dependence structure, as different copula characterizes different dependence structures. Therefore, the candidate copulas describing negative correlation should be sieved to match the dependence structure in the original sample. For this case, the Gaussian copula, Plackett copula, Frank copula, and No. 16 copula are specifically selected. Li et al. [22] and Zhang et al. [7] presented that these four copulas can model negative dependences. The values of correlation coefficients between obtained equivalent sample can cover the interval $(-1,0)$. However, the Gaussian and Plackett copulas belong to elliptical and Plackett copula families, respectively. The Frank and No. 16 copulas are commonly used Archimedean 


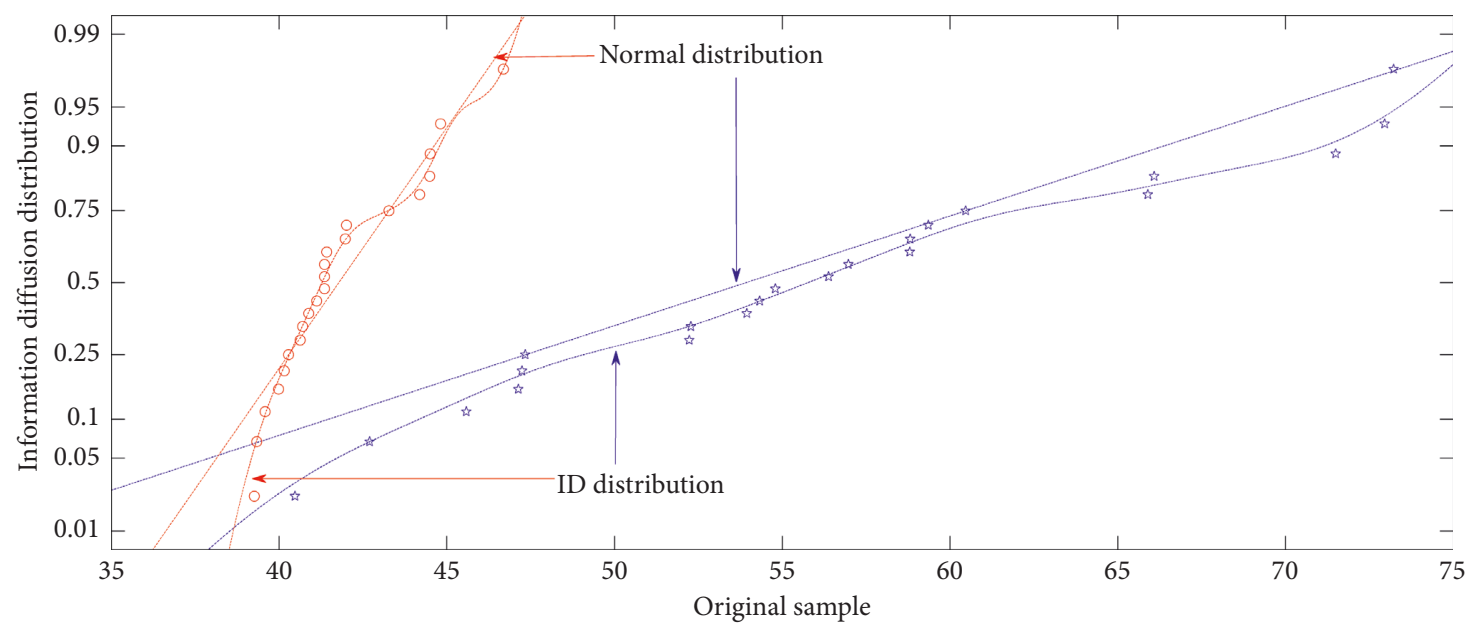

Figure 5: Probability plots of $c$ and $\varphi$ associated with ID and normal distributions.

TABLE 3: Common probability distribution functions of parameter.

\begin{tabular}{|c|c|c|c|}
\hline Type & PDF & $\mathrm{CDF}$ & Remark \\
\hline $\begin{array}{l}\text { Truncated } \\
\text { normal }\end{array}$ & $(\Phi((x-p) / q)) /[1-\Phi((0-p) / q)]$ & $\begin{array}{c}{[\Phi((x-p) / q)-\Phi((0-p) / q)] /} \\
{[1-\Phi((0-p) / q)]}\end{array}$ & $\begin{array}{l}p=\mu \\
q=\sigma\end{array}$ \\
\hline Lognormal & $((1) /(\sqrt{2 \pi} q x)) \exp \left[-(1 / 2)((\ln (x)-p) / q)^{2}\right]$ & $\Phi((\ln (x)-p) / q)$ & $\begin{array}{c}p=\ln \left[\mu /\left(\sqrt{\left(1+\sigma^{2}\right) / \mu^{2}}\right)\right] \\
q=\sqrt{\ln \left(1+\sigma^{2} / \mu^{2}\right)}\end{array}$ \\
\hline Gumbel & $\begin{array}{c}(q \exp \{-1(x-p)-\exp [-q(x-p)]\}) / \\
1-\exp [-\exp (p q)]\end{array}$ & $\begin{array}{c}(\exp \{-\exp [-q(x-p)]\} \\
-\exp [-\exp (p q)]) /(1-\exp [-\exp (p q)])\end{array}$ & $\begin{array}{c}p=(\mu-0.5772) / q \\
q=1.2825 / \sigma\end{array}$ \\
\hline Weibull & $(q / p)(x / p)^{q-1} \exp \left[-(x / p)^{q}\right]$ & $1-\exp \left[-(x / p)^{q}\right]$ & $\begin{array}{c}\mu=p \Gamma((1+1) / q) \\
\sigma=p \sqrt{\Gamma((1+2) / q)-\Gamma^{2}((1+1) / q)}\end{array}$ \\
\hline
\end{tabular}

TABLE 4: Goodness-of-fit test for different marginal distributions.

\begin{tabular}{lcccccccc}
\hline Distribution type & \multicolumn{2}{c}{$c$} & & & \multicolumn{2}{c}{$\varphi$} & \\
& $\alpha$ & $D_{n, \alpha}$ & $D_{n}$ & AIC & $\alpha$ & $D_{n, \alpha}$ & $D_{n}$ & AIC \\
\hline Information diffusion & 0.05 & 0.2809 & $\mathbf{0 . 0 9 7 5}$ & $\mathbf{1 5 4 . 0 9 4 4}$ & 0.05 & 0.2809 & $\mathbf{0 . 0 7 4 9}$ & $\mathbf{8 1 . 1 3 4 2}$ \\
Truncated normal & 0.05 & 0.2809 & 0.2083 & 164.1152 & 0.05 & 0.2809 & 0.1018 & 96.4004 \\
Lognormal & 0.05 & 0.2809 & 0.1991 & 163.5509 & 0.05 & 0.2809 & 0.1040 & 95.6181 \\
Gumbel & 0.05 & 0.2809 & 0.1439 & 164.7073 & 0.05 & 0.2809 & 0.1231 & 91.9265 \\
Weibull & 0.05 & 0.2809 & 0.2667 & 165.7791 & 0.05 & 0.2809 & 0.1302 & 107.8857 \\
\hline
\end{tabular}

copulas. Moreover, No. 16 copula is approximately symmetric in case of a strongly negative correlation. The other ones are symmetric copulas. Such properties are very suitable for modeling the dependence structure between $c$ and $\varphi$. The aforementioned copulas are summarized in Table 5.

By using equations (5) and (6), the parameter $\rho$ or $\theta$ could be computed, as listed in column 4 of Table 6 . Associated with the copula formulas in Table 5 , corresponding bivariate joint distributions are constructed. For visualization and comparison, 500 random pairs $\left(U_{1}, U_{2}\right)$ in rank space are produced by the aforementioned copula functions. These pairs would then be fed back to their base distributions to predict the equivalent samples. The scatter plots are successively portrayed in Figure 8, corresponding to the Gaussian, Plackett, Frank, and No. 16 copulas. It can be observed that all candidate copulas can cater to the symmetry along the diagonal line. Specially, Figure 8(a), representing Gaussian copula, consists with this behavior more remarkably than the other three subfigures. Table 5 tabulates the results of goodness-of-fit test by equations (7) and (9). It is clear that the Gaussian copula has the minimum SED and AIC value simultaneously, also indicating that the Gaussian copula is the most probable function for matching the dependence structure.

Additionally, equivalent samples can be obtained by the already determined marginal distributions of $c$ and $\varphi$. As analyzed earlier, ID distribution has been demonstrated to have the best fit. Therefore, with the inverse function of information diffusion, 500 random pairs $\left(U_{1}, U_{2}\right)$ are transformed back into the original units. Sequentially, four corresponding equivalent samples are obtained. The scatter plots (open circle in blue) are portrayed in Figure 9, along 


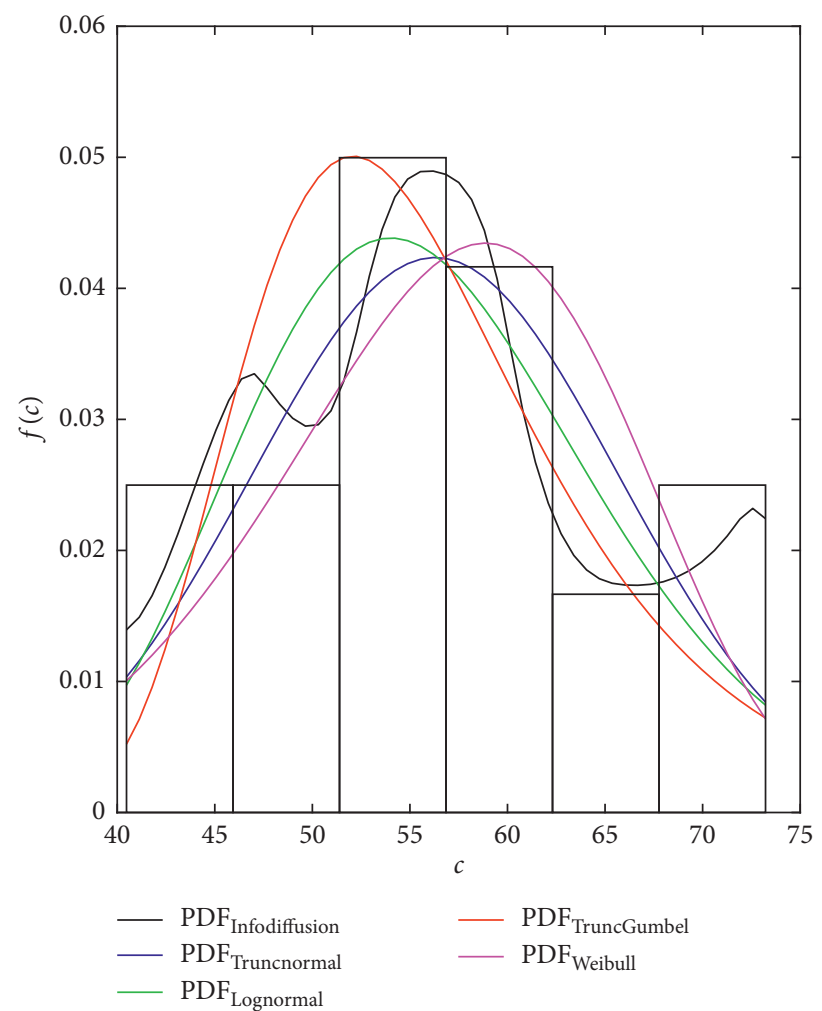

(a)

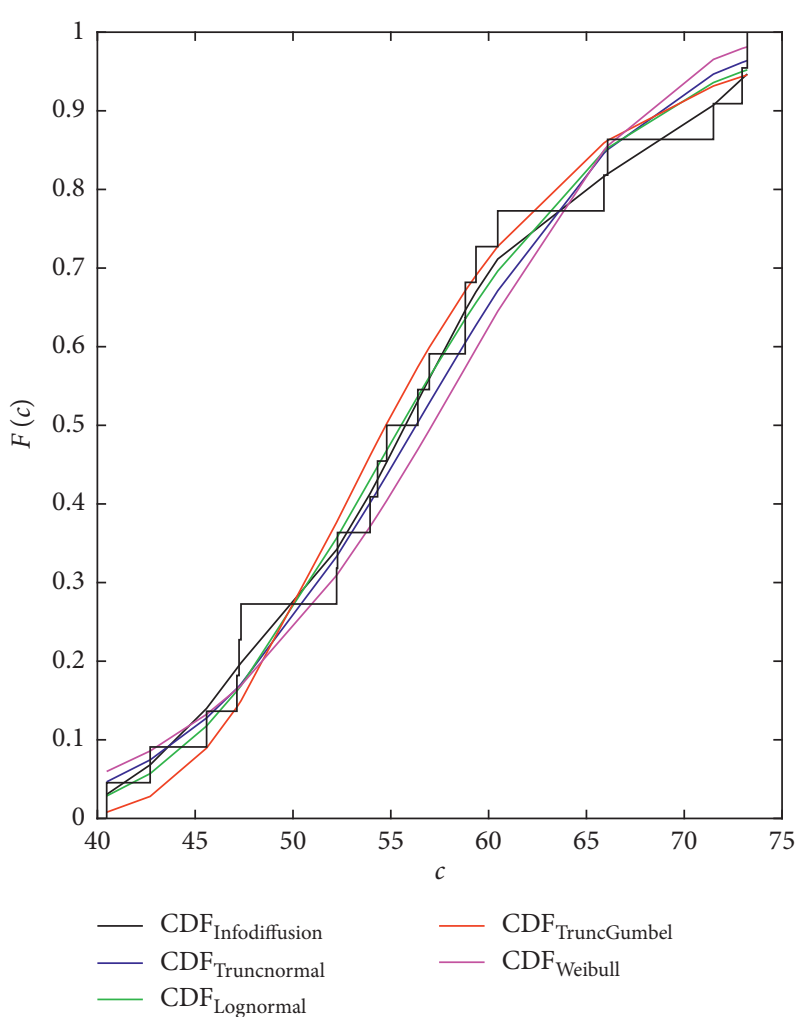

(b)

FIgURE 6: PDFs and CDFs of $c$ under different marginal distribution types.

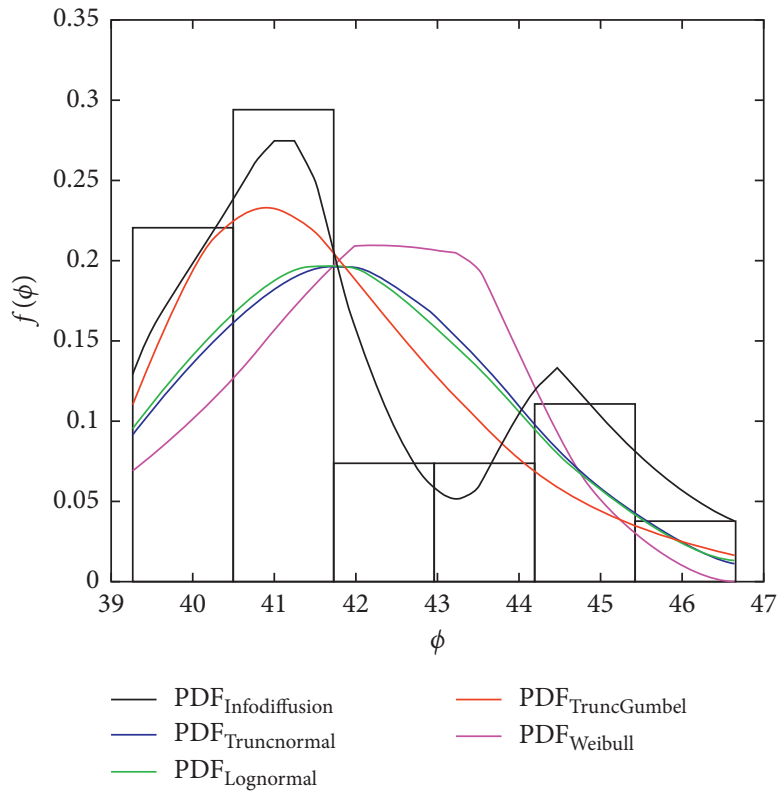

(a)

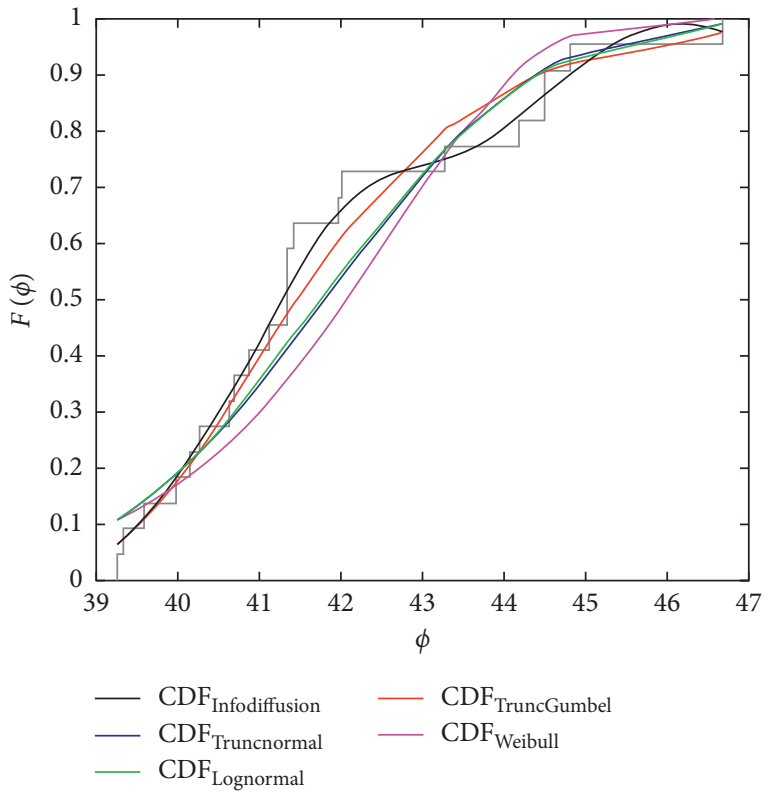

(b)

FIgURe 7: PDFs and CDFs of $\varphi$ under different marginal distribution types.

with the original data (solid circle in red). It is noticeable that the equivalent sample in Figure 9(a), reproduced by the Gaussian copula, holds a more concentrated distribution and covers the measured pairs better. In contrast, the others are relatively discrete, especially No. 16 copula. Therefore, the Gaussian copula captures the actual observation better than the other three cases. This can be further substantiated from the statistics of equivalent samples and AIC test, as 
Table 5: Copulas selected in this study.

\begin{tabular}{lccc}
\hline Copula type & $C(u, v ; \theta)$ & $c(u, v ; \theta)$ & $\theta$ \\
\hline Gaussian & $\Phi_{\theta}\left(\Phi^{-1}(u), \Phi^{-1}(v)\right)$ & $\Phi\left(\left(\Phi^{-1}\left(u_{2}\right)-\theta \Phi^{-1}\left(u_{1}\right)\right) /\left(\sqrt{1-\theta^{2}}\right)\right)$ & $(1 / 2)-\left(\left(1+(\theta-1) u_{1}-(\theta+1) u_{2}\right) /\right.$ \\
Plackett & $\left(S-\sqrt{S^{2}-4 u v \theta(\theta-1)}\right) /(2(\theta-1))$, & $\left.\left(2\left\{\left[1+(\theta-1)\left(u_{1}+u_{2}\right)\right]-4 u_{1} u_{2} \theta(\theta-1)\right\}\right)\right)$ & $(0,+\infty) \backslash\{1\}$ \\
Frank & $S=1+(\theta-1)(u+v)$ & $\left(e^{-\theta u}\left(e^{-\theta v}-1\right)\right) /\left(\left(e^{-\theta}-1\right)+\left(e^{-\theta u}-1\right)\left(e^{-\theta v}-1\right)\right)$ & $(-\infty,+\infty) \backslash\{0\}$ \\
No. 16 & $-(1 / \theta) \ln \left[1+\left(\left(\left(e^{-\theta u}-1\right)\left(e^{-\theta v}-1\right)\right) /\left(e^{-\theta}-1\right)\right)\right]$ & $(1 / 2)\left(1+\left(\theta / u^{2}\right)\right)\left[1+S\left(S^{2}+4 \theta\right)^{-(1 / 2)}\right]$ & {$[0,+\infty)$} \\
& $S=u+v-1-\theta((1 / u)+(1 / v)-1)$ & $S=u+v-1-\theta((1 / u)+(1 / v)-1)$ &
\end{tabular}

TABLE 6: Related parameters of copulas for $c$ and $\varphi$.

\begin{tabular}{lccccccccc}
\hline Copula type & Pearson & $\tau_{\mathrm{o}}$ & $\Theta$ & $\tau_{U}$ & $P_{U}$ & $\tau_{X}$ & $P_{X}$ & SED & AIC \\
\hline Gaussian & & & -0.9432 & -0.7764 & -0.9332 & -0.7764 & -0.8887 & $\mathbf{0 . 0 1 1 2}$ & $-\mathbf{4 3 . 8 9 8}$ \\
Plackett & & & 0.0103 & -0.7865 & -0.9177 & -0.7865 & -0.8459 & 0.3551 & -40.115 \\
Frank & -0.8767 & -0.7843 & -16.7225 & -0.7817 & -0.9382 & -0.7817 & -0.8863 & 0.0139 & -37.198 \\
No. 16 & & & 0.0017 & -0.7896 & -0.9319 & -0.7896 & -0.8760 & 0.0130 & -35.735 \\
\hline
\end{tabular}

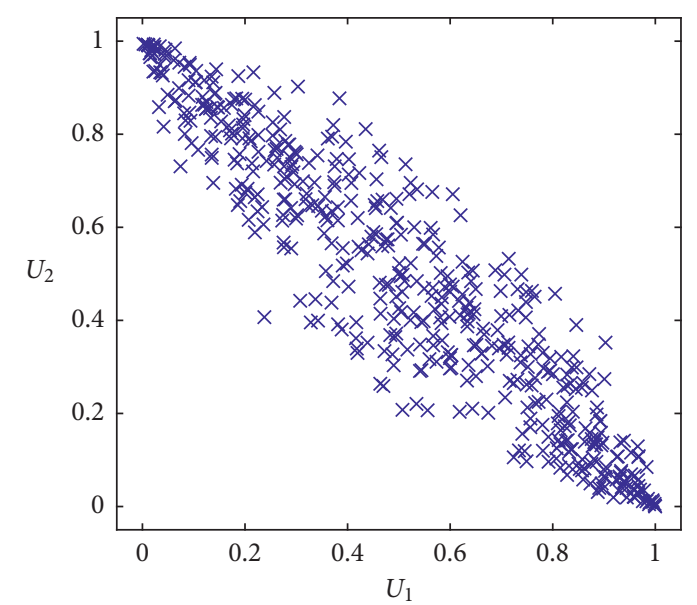

(a)

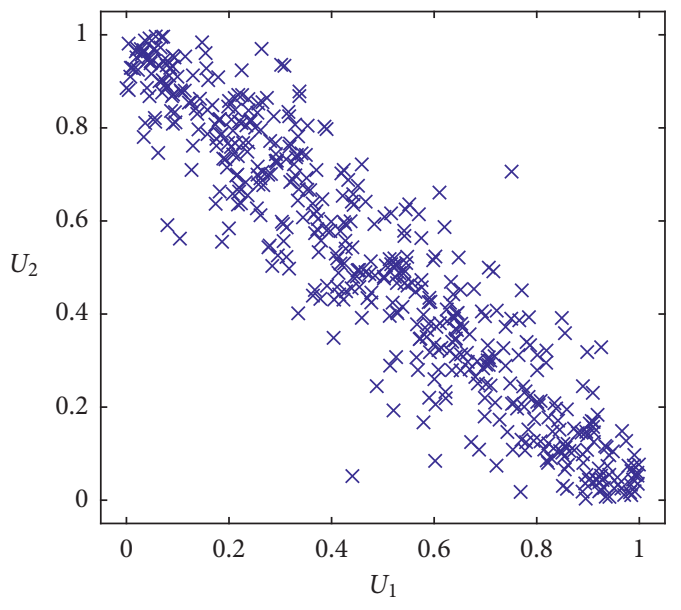

(c)

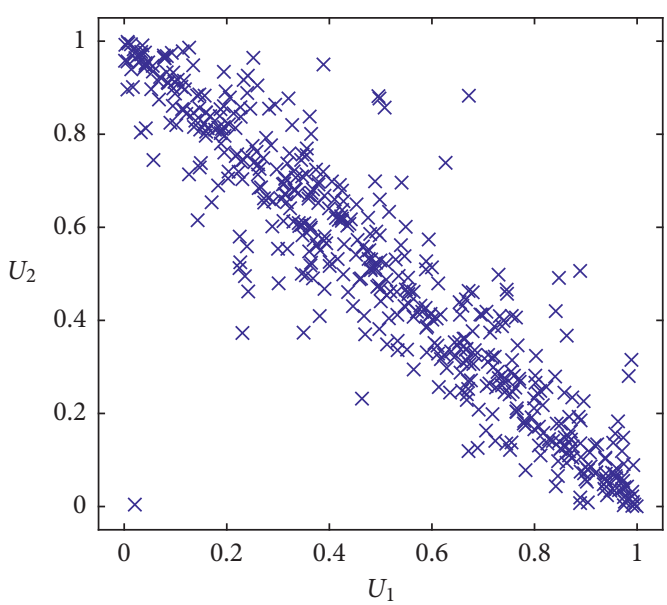

(b)

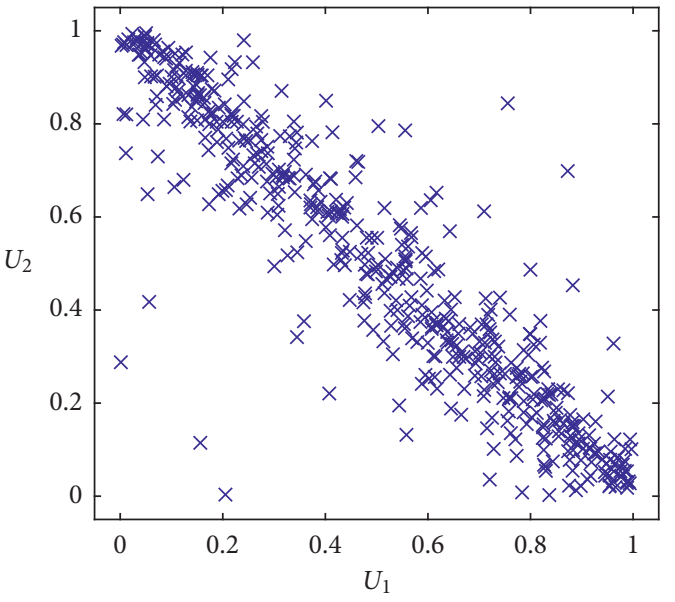

(d)

FIGURE 8: Scatter plots of simulated pairs using different copulas. (a) Gaussian copula. (b) Plackett copula. (c) Frank copula. (d) No. 16 copula.

shown in Table 7. It can be seen in Table 7 that the statistics corresponding to different copulas are almost similar and close to those of observed data. However, Gaussian copula coupling ID distribution can reproduce the statistics of original data with the minimum AIC values, indicating a relatively high accuracy.

Notably, Gaussian copula has been validated to be the best candidate function to characterize the underlying 


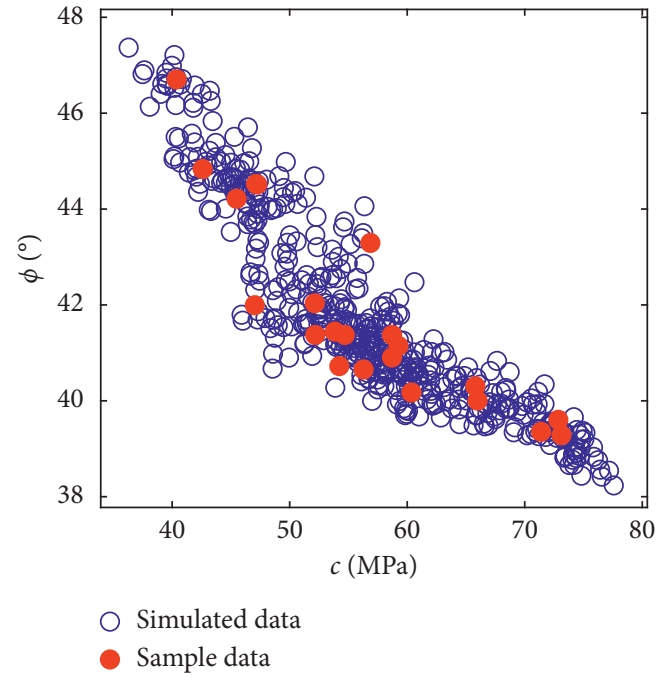

(a)

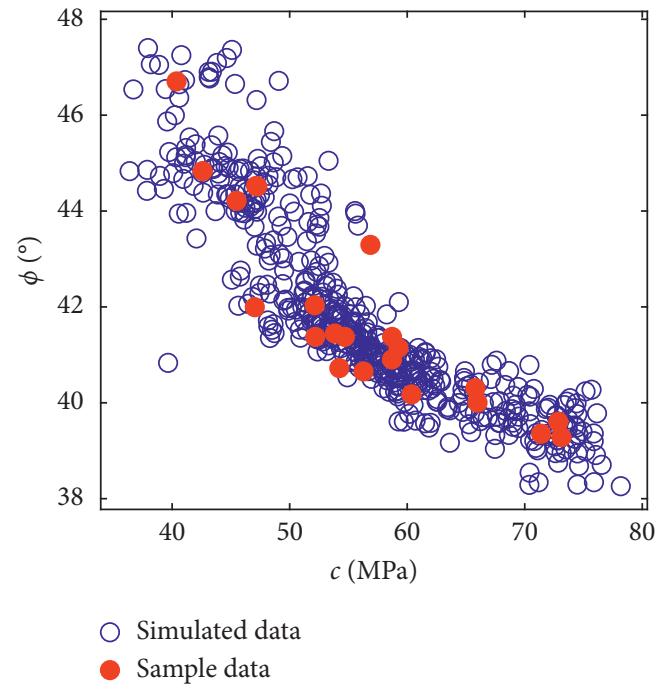

(c)

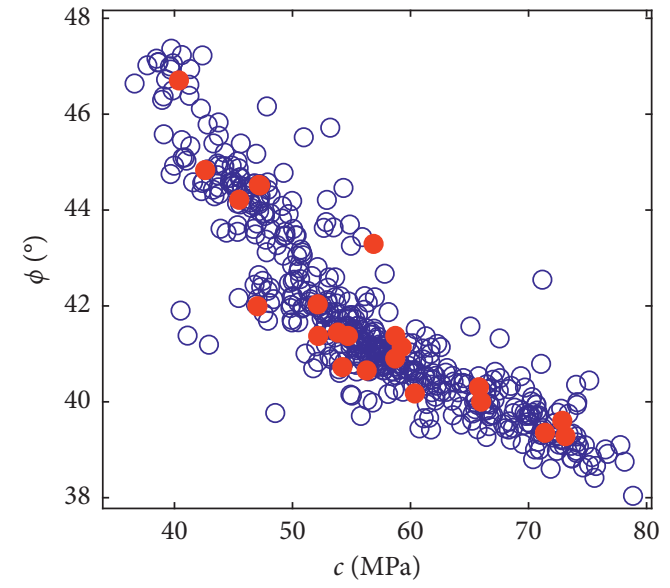

O Simulated data

- Sample data

(b)

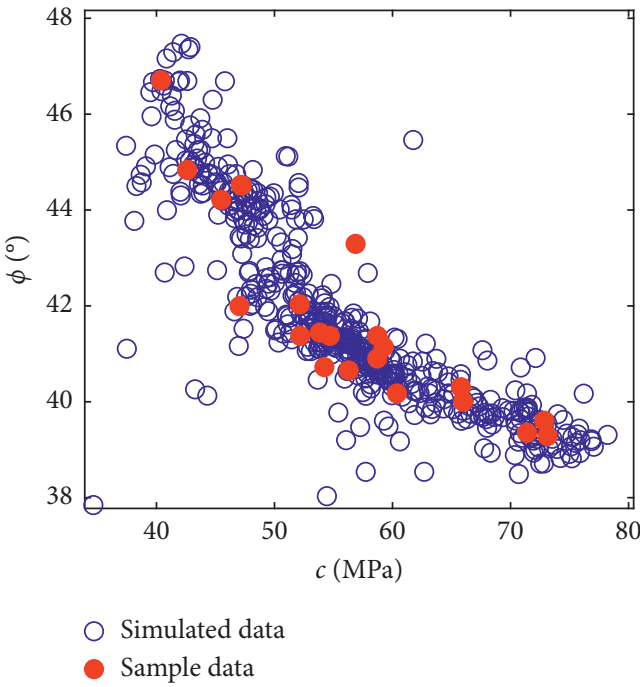

(d)

FIgURE 9: Scatter plots of equivalent samples generated by different copulas coupling ID margins. (a) Gaussian copula. (b) Plackett copula. (c) Frank copula. (d) No. 16 copula.

Table 7: Parameters of different marginal distributions coupling Gaussian copula.

\begin{tabular}{|c|c|c|c|c|c|c|c|c|}
\hline \multirow{2}{*}{ Copula function } & \multicolumn{4}{|c|}{$C$} & \multicolumn{4}{|c|}{$\varphi$} \\
\hline & $\mu_{c}$ & $\sigma_{c}$ & $\operatorname{cov}_{c}$ & AIC & $\mu_{\varphi}$ & $\sigma_{\varphi}$ & $\operatorname{cov}_{\varphi}$ & AIC \\
\hline Gaussian & 56.03 & 9.291 & 0.1658 & 3560.24 & 41.78 & 2.029 & 0.0486 & 1915.84 \\
\hline Plackett & 56.79 & 9.437 & 0.1662 & 3571.15 & 41.72 & 2.056 & 0.0493 & 1938.89 \\
\hline Frank & 56.53 & 9.478 & 0.1677 & 3589.63 & 41.77 & 2.028 & 0.0486 & 1951.95 \\
\hline No. 16 & 56.82 & 9.524 & 0.1676 & 3605.30 & 41.69 & 1.996 & 0.0479 & 1932.66 \\
\hline
\end{tabular}

dependence structure among the insite data. However, it is only responsible for the site-specific data in this study. Whether it holds in other cases should be reanalyzed.

\subsection{Backward Analysis for Marginal Distribution of Equiv-} alent Sample. As previously discussed, Gaussian copula is the most probable bivariate joint distribution of the original pair $(c, \varphi)$. Then, via information diffusion approach, the equivalent sample can be accurately simulated, as shown in Figure 9(a). Nevertheless, after encountering multiple mathematical treatments, whether the equivalent sample holds consistency with original data needs to be further validated. To this end, a backward analysis for the proposed model is desirable.

For comparison, the aforementioned conventional marginal distributions are implemented simultaneously coupling the four candidate copulas. Figures 10-13 portray 


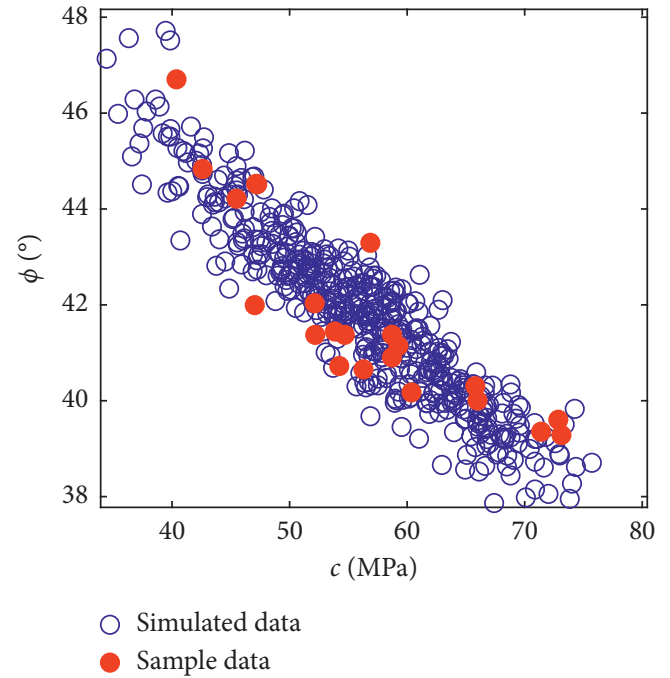

(a)

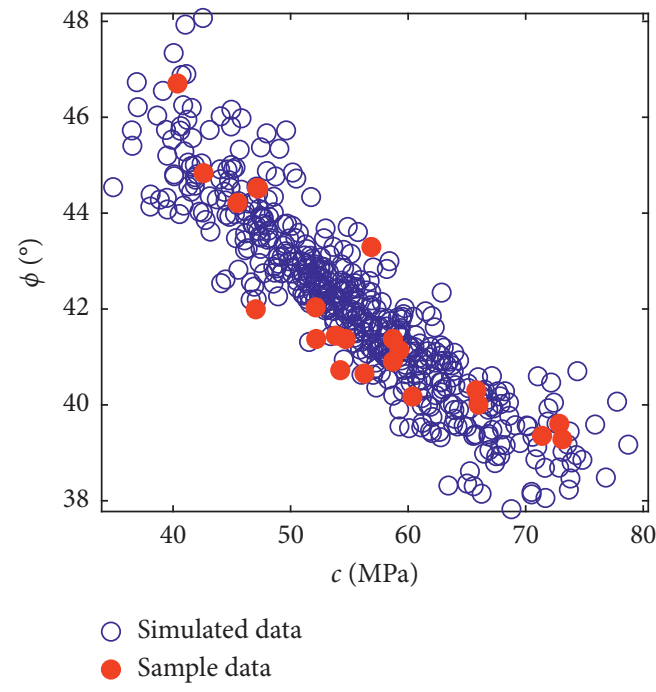

(c)

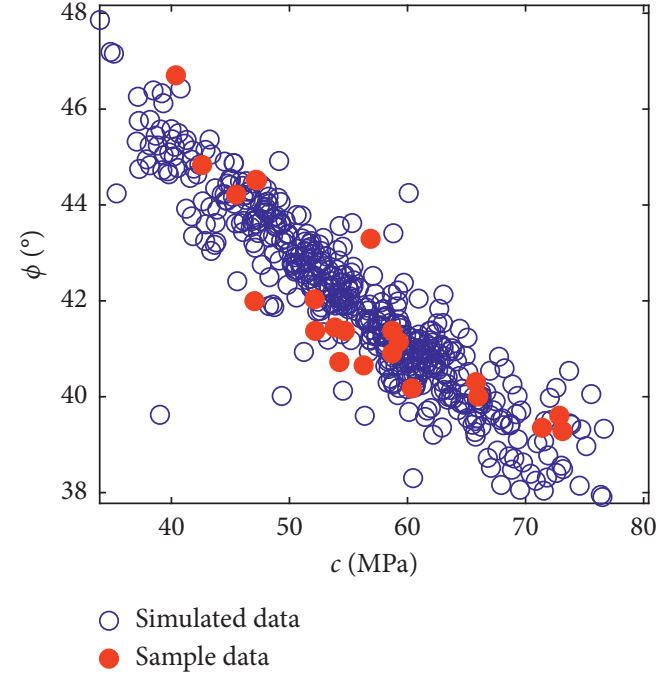

(b)

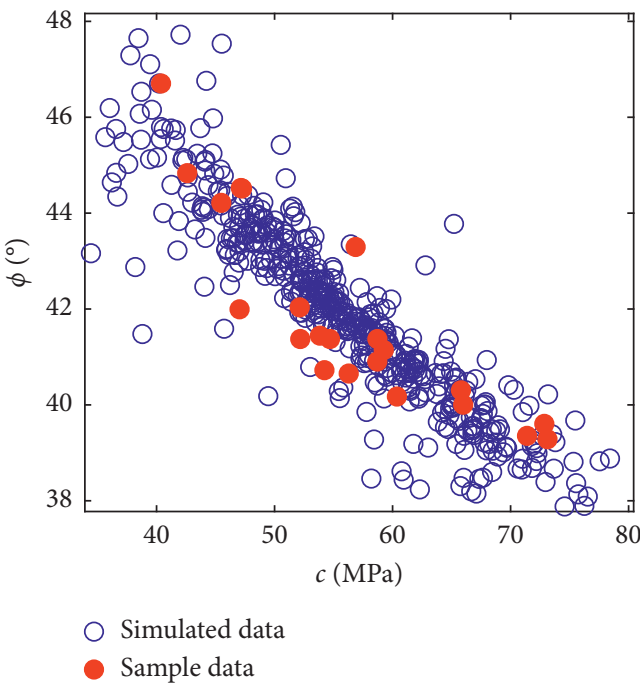

(d)

FIgURE 10: Scatter plots of equivalent samples generated by different copulas coupling Truncnormal margins. (a) Gaussian copula. (b) Plackett copula. (c) Frank copula. (d) No. 16 copula.

the scatter plots of equivalent samples yielded by four candidate copulas coupling different conventional margins. It can be clearly observed that there are significant differences in dependence structures associated with the four candidate copulas even though the same margins and correlation coefficients are utilized. These are in accordance with the studies of Li et al. [22] and Zhang et al. [7]. It can also be seen that the equivalent samples corresponding to different margins have a larger discrepancy. For instance, associated with the first subplots of Figures 9(a)-13(a), the same dependence structure is modeled by Gaussian. However, the shapes of scatter plots differ noticeably from each other. This is mainly due to different marginal distribution structures of $c$ and $\varphi$. Next, some deviations in discrete degree can be observed from the scatter distributions. In Figures 10-13, four subplots corresponding to Gaussian copula illuminate that the scatters become concentrated gradually from both tails to the middle. Some original points distribute along the edges of distribution domains, or even disperse beyond these domains, especially in Figure 13(a). However, in terms of Figure 9(a), concentrated distribution and discrete distribution of scatters appear alternately. This behavior obviously follows the true distribution state owing to heterogeneity of rock and soil. Furthermore, the equivalent samples in Figure 9(a) have the highest coverage rate and have similar scatterings as initial data. Such a difference is dictated by copula function, marginal distributions, and the interaction between them. Similar results can be drawn by comparing other subplots.

The corresponding distribution statistics of equivalent samples and AIC values of candidate distributions are computed and tabulated in Table 8. Comparing Table 7 with Table 8 , it is evident that no matter how the copulas and margins be incorporated, the means, standard deviations, and coefficients of variation of equivalent samples barely 

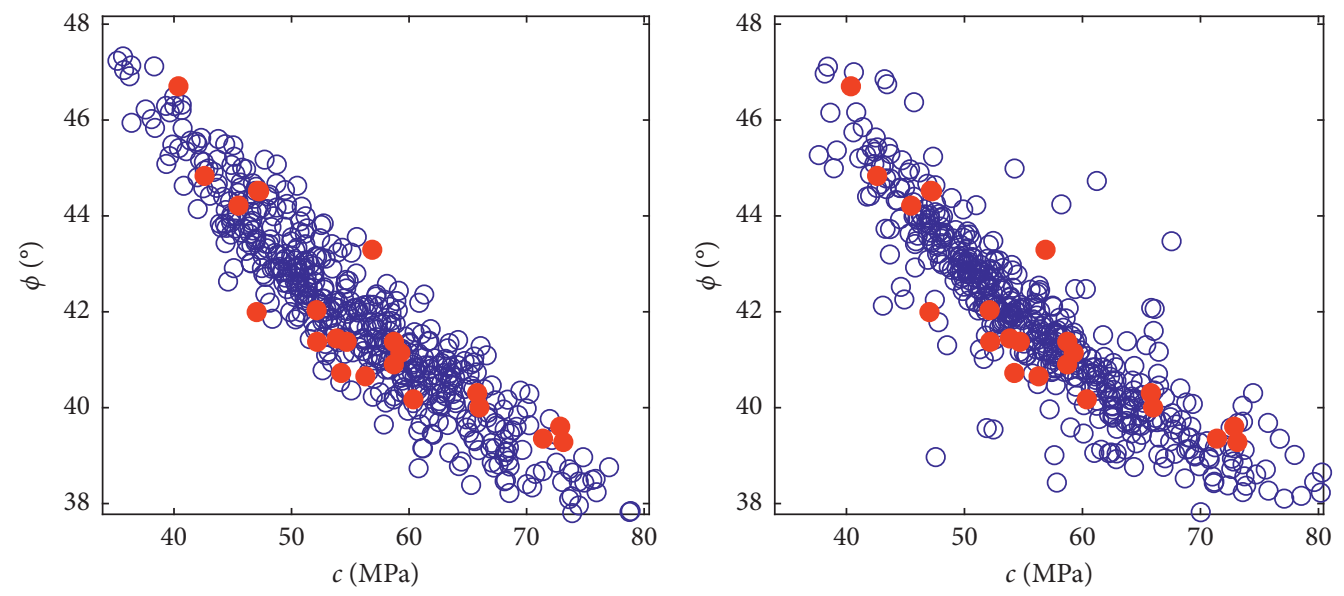

O Simulated data

Sample data

(a)

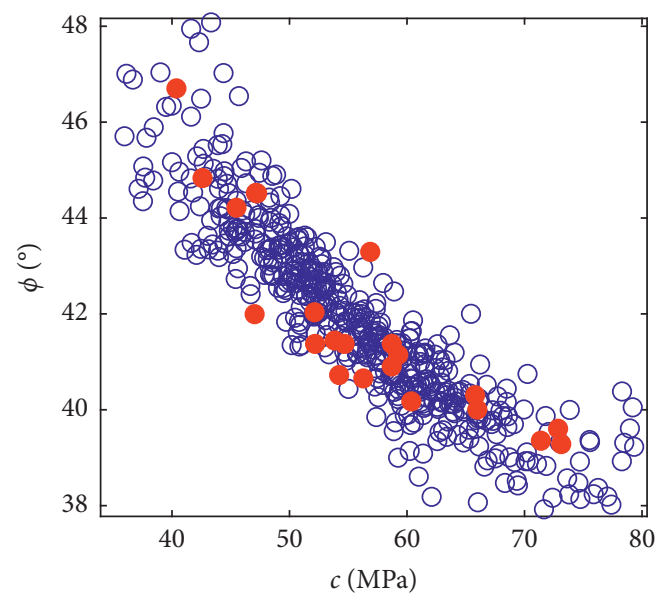

Simulated data

- Sample data

(b)

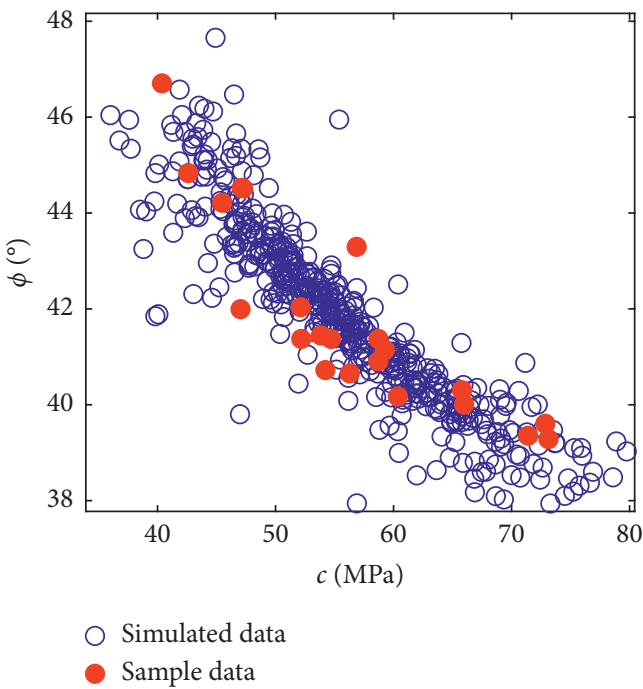

(d)

FiguRE 11: Scatter plots of equivalent samples generated by different copulas coupling Lognormal margins. (a) Gaussian copula. (b) Plackett copula. (c) Frank copula. (d) No. 16 copula.

change and basically cater to those of observed data. All the conventional margins and ID distribution can reproduce the statistics of original database with a high enough accuracy. However, the AIC values vary significantly in a comparative sense. Assuredly, coupling similar copula function, different marginal distributions have marginal impacts on estimation of equivalent samples. It is clear that Gaussian copula coupling ID distribution has the minimum AIC values, indicating its best approximation and consistency.

From the above analysis, it can be derived that Gaussian copula coupling ID distribution not only can capture the broad shape of initial sample better but also gives more reasonable overall result.

For a more intuitive comparison, Figures 14 and 15 give the univariate PDFs and CDFs of equivalent samples reproduced by Gaussian copula coupling different margins, along with the histogram of site-specific data. It is noticeable that PDF plots of $c$ and $\varphi$ recreated by ID technique retain fluctuating changes with multiple peaks, coinciding with the tendency and volatility of their distribution histograms. Contrarily, conventional cases cannot represent the volatility noticeably. It can also be observed that the CDF plots associated with ID distribution are closest to the empirical distribution, further exemplifying the superiority of the proposed approach.

Consequently, all the above results demonstrate that marginal distribution has a considerable impact on the parameter estimation. Neglecting the base distribution and the property of random volatility might lead to unbiased estimation and inevitable uncertainty. Fortunately, ID approach provides a robust analysis on the base distribution of individual shear strength parameter. It enables the information extraction and propagation from initial data efficiently. Particularly, it assists in capturing random 

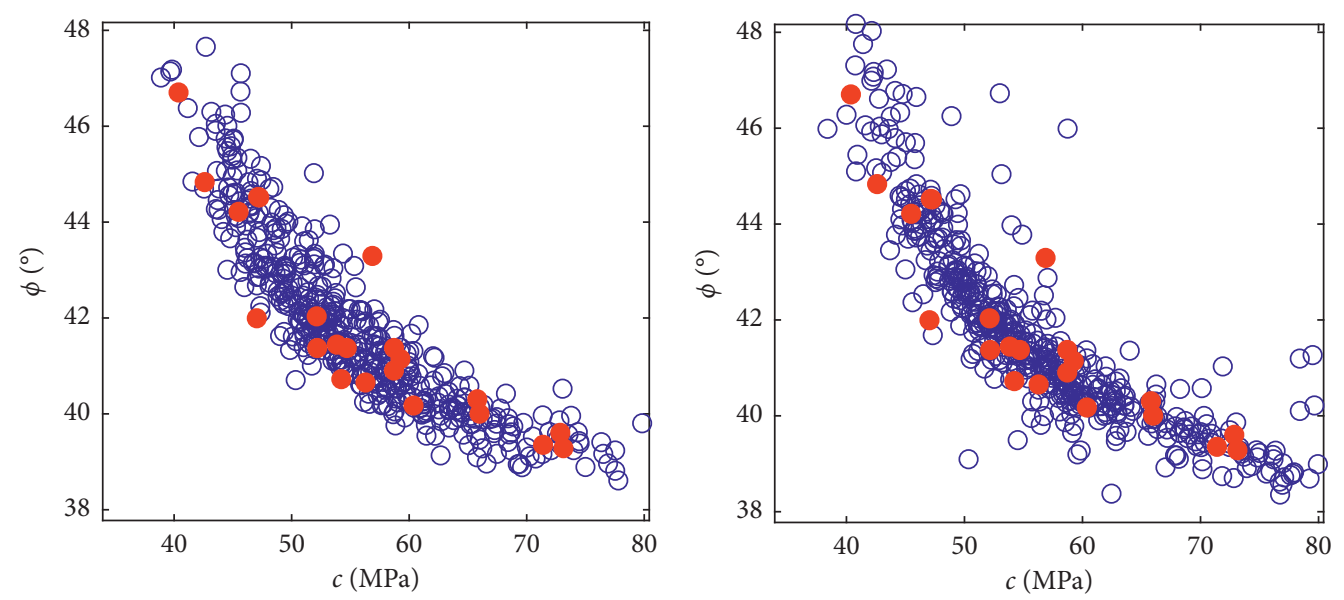

O Simulated data

Simulated data

- Sample data

- Sample data

(a)
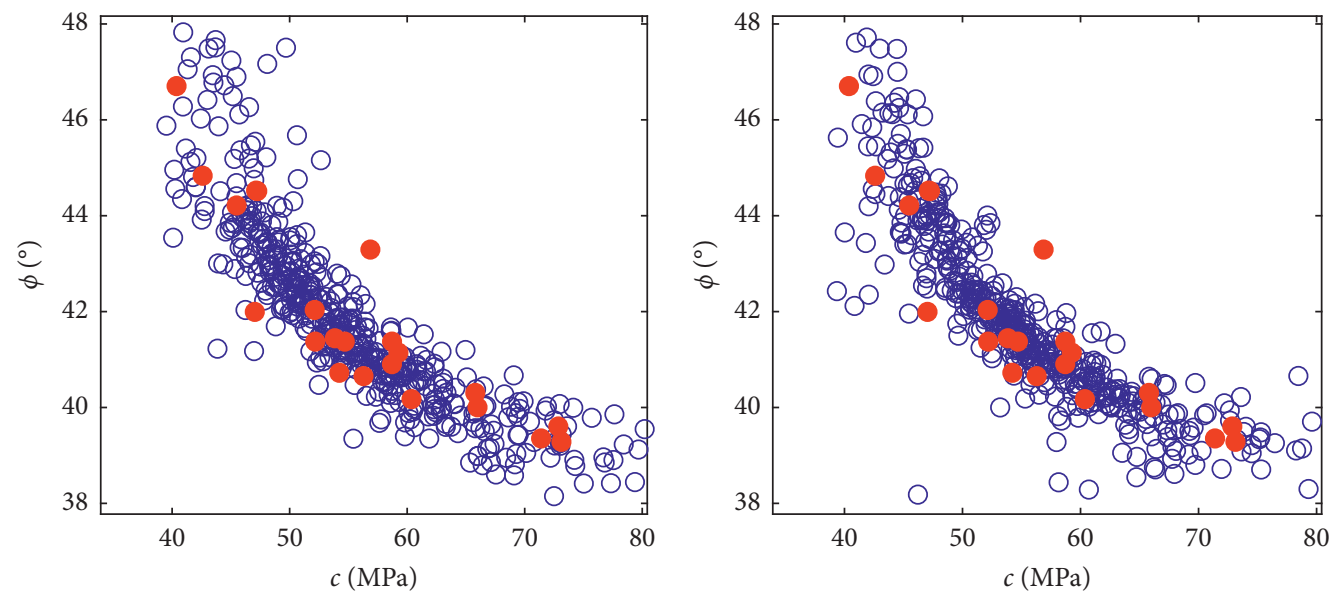

O Simulated data

Sample data

Simulated data

- Sample data

(c)

(d)

FIGURE 12: Scatter plots of equivalent samples generated by different copulas coupling Tunnc Gumbel margins. (a) Gaussian copula. (b) Plackett copula. (c) Frank copula. (d) No. 16 copula.

volatility of shear strength parameters, which cannot be ignored in reality.

\section{Conclusions}

This paper developed an information diffusion technique to estimate the marginal distribution of shear strength parameters, in conjunction with the copula theory employed to model the joint distribution between $c$ and $\varphi$ using a small sample. The proposed approach was illustrated and validated by actual observations from the laboratory test for Bukit Timah area in Singapore. Several outstanding conclusions can be drawn from this study:

(1) Under incomplete probability information, conventional distributions cannot describe properties of the actual distribution sufficiently, especially random volatility. In contrast, information diffusion technique is capable of incomplete probabilistic information. Via capturing and diffusing the internal information, marginal distribution can be credibly expressed. In comparison with the conventional marginal distributions, the proposed approach result in a more reasonable estimate of shear strength parameters and characterize the random volatility better, which make sure that marginal distribution can extremely reflect actual state.

(2) Mathematically, copula theory has been extended to model the underlying interdependency between $c$ and $\varphi$, and then the equivalent sample has been obtained by coupling information diffusion distribution. The results show that the Gaussian copula has the minimum SED and AIC value simultaneously, indicating that it is the most probable function for matching the dependence structure between the site-specific data. 

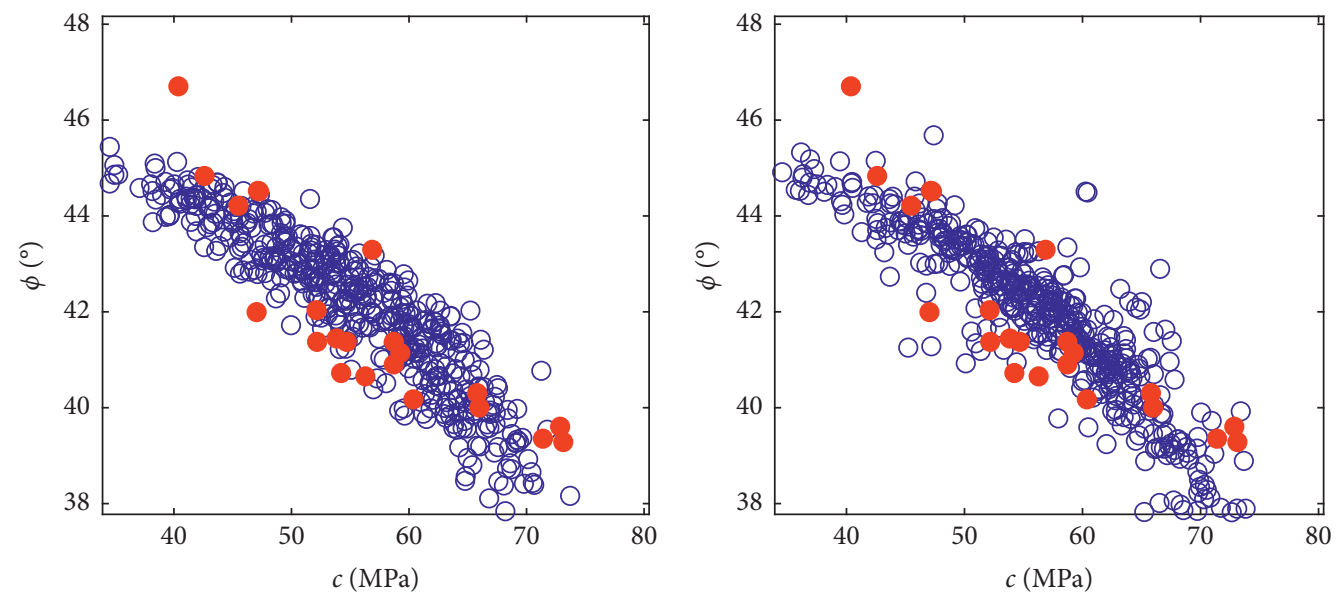

O Simulated data

O Simulated data

Sample data

(a)

(b)
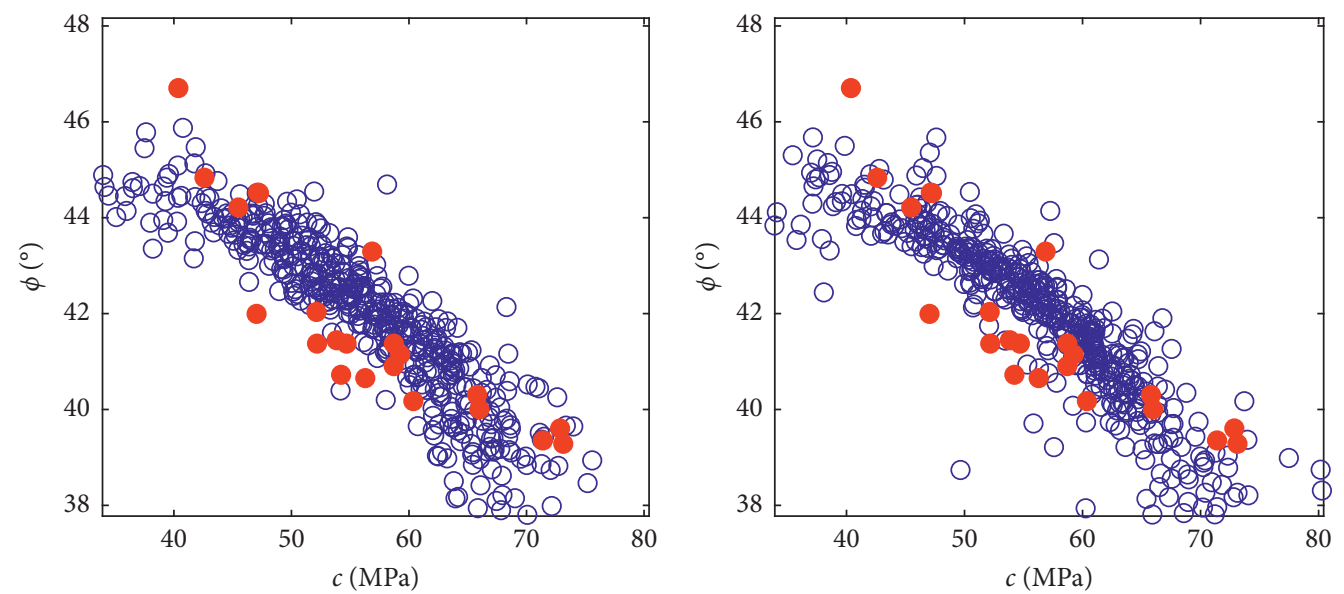

Simulated data

O Simulated data

- Sample data

- Sample data

(c)

(d)

FIGURE 13: Scatter plots of equivalent samples generated by different copulas coupling Weibull margins. (a) Gaussian copula. (b) Plackett copula. (c) Frank copula. (d) No. 16 copula.

TABLE 8: Statistics and AIC values of equivalent samples associated with four candidate copulas coupling different conventional margins.

\begin{tabular}{|c|c|c|c|c|c|c|c|c|c|}
\hline \multirow{2}{*}{ Margin } & \multirow{2}{*}{ Copula } & \multicolumn{4}{|c|}{$C$} & \multicolumn{4}{|c|}{$\varphi$} \\
\hline & & $\mu_{c}$ & $\sigma_{c}$ & $\operatorname{cov}_{c}$ & AIC & $\mu_{\varphi}$ & $\sigma_{\varphi}$ & $\operatorname{cov}_{\varphi}$ & AIC \\
\hline \multirow{4}{*}{ Truncnormal } & Gaussian & 56.09 & 9.419 & 0.1679 & 3662.71 & 41.80 & 2.021 & 0.0484 & 2123.74 \\
\hline & Plackett & 56.89 & 9.419 & 0.1656 & 3662.71 & 41.72 & 2.040 & 0.0489 & 2133.03 \\
\hline & Frank & 56.57 & 9.419 & 0.1665 & 3662.71 & 41.76 & 2.004 & 0.0480 & 2115.23 \\
\hline & No. 16 & 56.79 & 9.419 & 0.1659 & 3662.71 & 41.69 & 1.986 & 0.0476 & 2106.13 \\
\hline \multirow{4}{*}{ Lognormal } & Gaussian & 56.09 & 9.455 & 0.1686 & 3638.42 & 41.80 & 2.019 & 0.0483 & 2122.25 \\
\hline & Plackett & 56.89 & 9.610 & 0.1689 & 3652.54 & 41.72 & 2.040 & 0.0489 & 2129.60 \\
\hline & Frank & 56.57 & 9.452 & 0.1671 & 3646.85 & 41.76 & 2.001 & 0.0479 & 2112.87 \\
\hline & No. 16 & 56.79 & 9.520 & 0.1676 & 3650.73 & 41.69 & 1.984 & 0.0476 & 2102.11 \\
\hline \multirow{4}{*}{ Gumbel } & Gaussian & 56.11 & 9.588 & 0.1709 & 3565.14 & 41.79 & 2.024 & 0.0484 & 2034.85 \\
\hline & Plackett & 56.90 & 9.831 & 0.1728 & 3593.99 & 41.73 & 2.042 & 0.0489 & 2030.89 \\
\hline & Frank & 56.56 & 9.466 & 0.1674 & 3582.21 & 41.75 & 1.958 & 0.0469 & 2020.97 \\
\hline & No. 16 & 56.78 & 9.657 & 0.1701 & 3590.05 & 41.69 & 1.960 & 0.0470 & 1998.74 \\
\hline \multirow{4}{*}{ Weibull } & Gaussian & 56.10 & 9.434 & 0.1682 & 3646.36 & 41.80 & 2.070 & 0.0495 & 2057.28 \\
\hline & Plackett & 56.88 & 9.257 & 0.1627 & 3633.73 & 41.71 & 2.046 & 0.0491 & 2078.69 \\
\hline & Frank & 56.57 & 9.386 & 0.1659 & 3638.12 & 41.77 & 2.007 & 0.0481 & 2053.89 \\
\hline & No. 16 & 56.78 & 9.345 & 0.1646 & 3634.3 & 41.70 & 1.981 & 0.0475 & 2055.81 \\
\hline
\end{tabular}




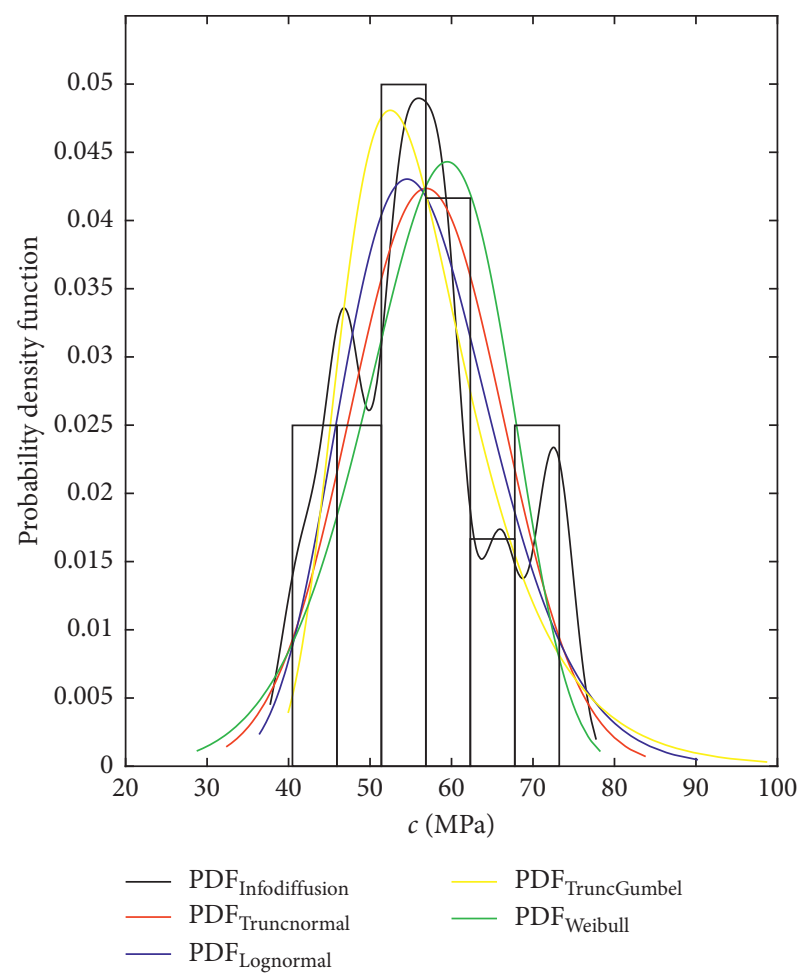

(a)

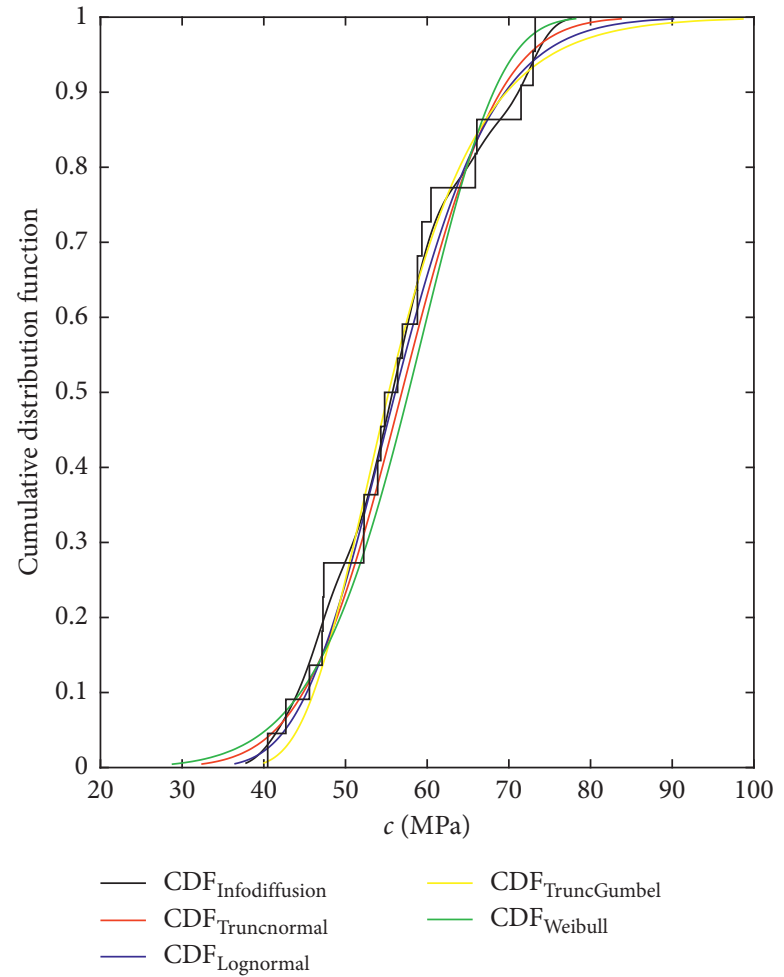

(b)

FIGURE 14: PDF and CDF plots of $c^{\prime}$ reproduced by Gaussian copula coupling different margins.

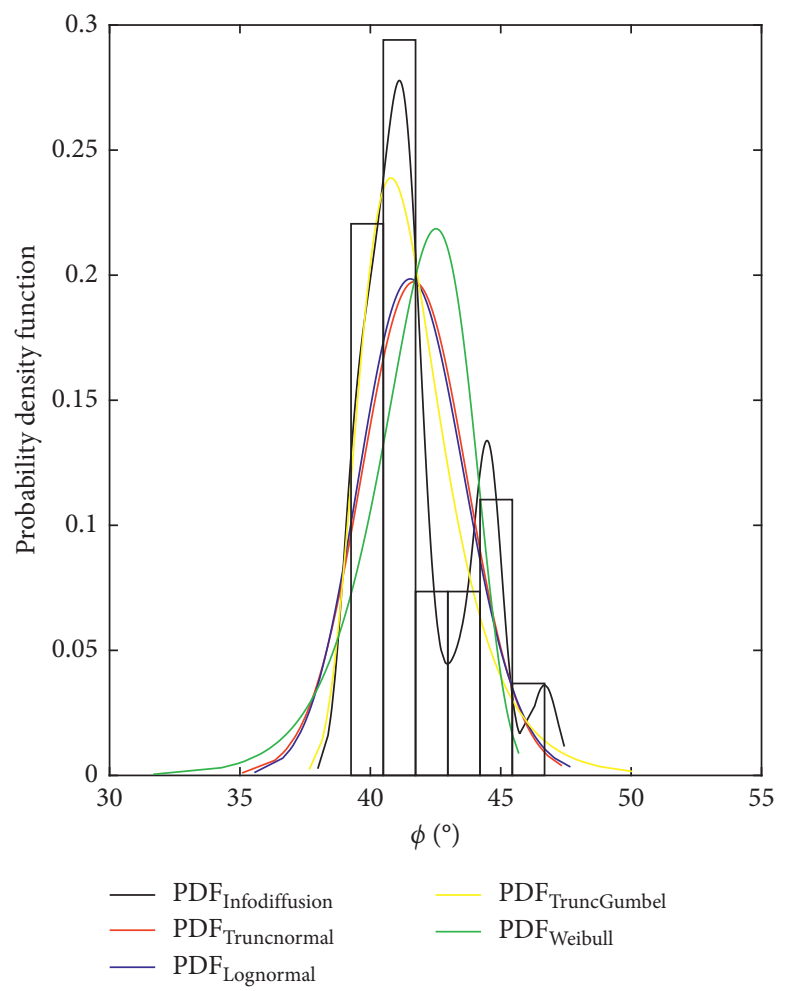

(a)

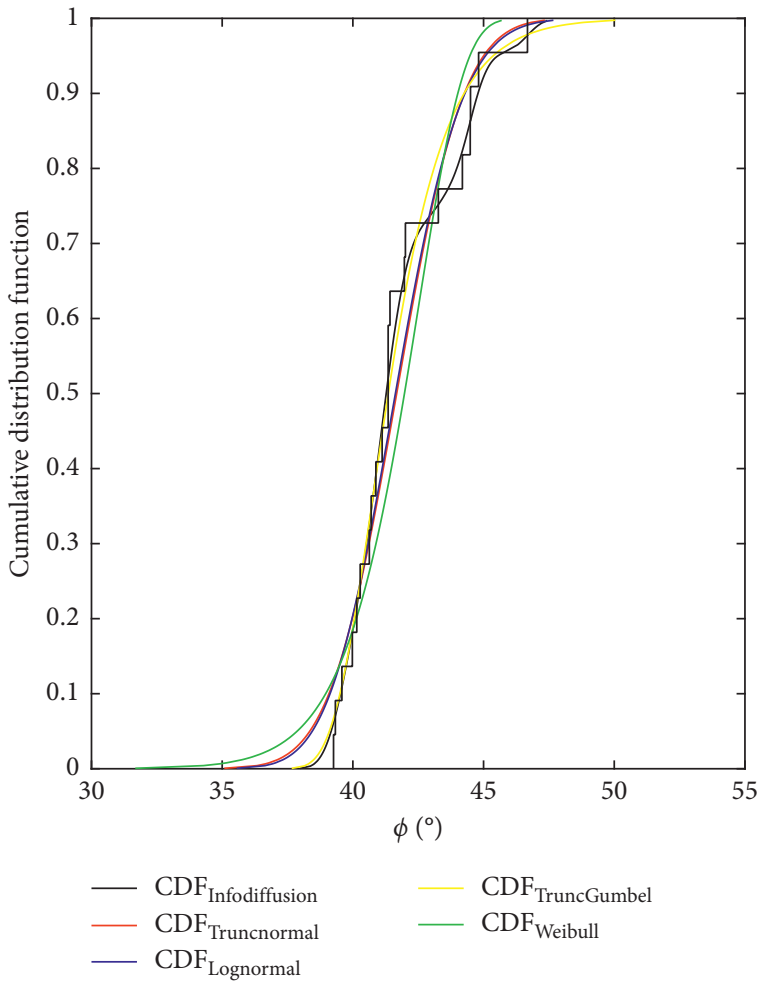

(b)

FIGURE 15: PDF and CDF plots of $\varphi^{\prime}$ reproduced by Gaussian copula coupling different margins. 
(3) The performances of the candidate copulas coupling conventional marginal distributions and ID distribution have been further discussed. In spite of identical copula function and correlation coefficients adopted to characterize the dependence structure, equivalent samples transformed by different margins exhibit major difference. As a result, Gaussian copula coupling ID distribution stands out attributed to minimum AIC values and optimal scatter distribution. The corresponding probability distributions of shear strength parameters validate the ability and accuracy of capturing random volatility of the proposed method.

\section{Data Availability}

Previously reported (original database of shear strength parameters) data were used to support this study and are available at http://www.rockmech.org/EN/Y2013/V32/I11/ 2225. This prior study is cited at relevant places within the text as reference [33].

\section{Conflicts of Interest}

The authors declare no conflicts of interest.

\section{Acknowledgments}

This research was supported by the State Key Laboratory of Safety and Health for Metal Mines under Grant no. 2016JSKSSYS-01, the National Natural Science Foundation of China under Grant no. 51609184, and the National Key Research and Development Program of China under Grant no. 2017YFC0804600.

\section{References}

[1] D. V. Griffiths, J. Huang, and G. A. Fenton, "Probabilistic infinite slope analysis," Computers and Geotechnics, vol. 38, no. 4, pp. 577-584, 2011.

[2] A. Ali, J. Huang, A. V. Lyamin et al., "Simplified quantitative risk assessment of rainfall-induced landslides modelled by infinite slopes," Engineering Geology, vol. 179, pp. 102-116, 2014.

[3] A. Johari and A. Mehrabani Lari, "System probabilistic model of rock slope stability considering correlated failure modes," Computers and Geotechnics, vol. 81, pp. 26-38, 2017.

[4] J. Ji, C. Zhang, Y. Gao, and J. Kodikara, "Effect of 2D spatial variability on slope reliability: a simplified FORM analysis," Geoscience Frontiers, vol. 9, no. 6, pp. 1631-1638, 2018.

[5] X.-S. Tang, D.-Q. Li, Y.-F. Chen, C.-B. Zhou, and L.-M. Zhang, "Improved knowledge-based clustered partitioning approach and its application to slope reliability analysis," Computers and Geotechnics, vol. 45, pp. 34-43, 2012.

[6] S.-H. Jiang and J. Huang, "Modeling of non-stationary random field of undrained shear strength of soil for slope reliability analysis," Soils and Foundations, vol. 58, no. 1, pp. 185-198, 2018.

[7] L. Zhang, D.-Q. Li, X.-S. Tang, Z.-J. Cao, and K.-K. Phoon, "Bayesian model comparison and characterization of bivariate distribution for shear strength parameters of soil," Computers and Geotechnics, vol. 95, pp. 110-118, 2018.
[8] F. Wang and H. Li, "Subset simulation for non-Gaussian dependent random variables given incomplete probability information," Structural Safety, vol. 67, pp. 105-115, 2017.

[9] P. Jolicoeur, "Interval estimation of the slope of the major Axis of a bivariate normal distribution in the case of a small sample," Biometrics, vol. 24, no. 3, pp. 679-682, 1968.

[10] I. Olkin and R. Liu, "A bivariate beta distribution," Statistics \& Probability Letters, vol. 62, no. 4, pp. 407-412, 2003.

[11] A. Nataf, "Détermination des distributions de probabilitédont les margessontdonnées," Comptes Rendus Hebdomadaires des Seances de l'Académie des Sciences, vol. 225, pp. 42-43, 1962.

[12] X. Z. Wu, "Trivariate analysis of soil ranking-correlated characteristics and its application to probabilistic stability assessments in geotechnical engineering problems," Soils and Foundations, vol. 53, no. 4, pp. 540-556, 2013.

[13] M. Motamedi and R. Y. Liang, "Probabilistic landslide hazard assessment using Copula modeling technique," Landslides, vol. 11, no. 4, pp. 565-573, 2014.

[14] G. K. Das, B. Hazra, A. Garg, and C. W. W. Ng, "Stochastic hydro-mechanical stability of vegetated slopes: an integrated copula based framework," Catena, vol. 160, pp. 124-133, 2018.

[15] E. W. Frees and E. A. Valdez, "Understanding relationships using copulas," North American Actuarial Journal, vol. 2, no. 1, pp. 1-25, 1998.

[16] S. Khajehei and H. Moradkhani, "Towards an improved ensemble precipitation forecast: a probabilistic postprocessing approach," Journal of Hydrology, vol. 546, pp. 476-489, 2017.

[17] G. K. Das, B. Hazra, A. Garg, and C. W. W. Ng, "Impact of hydrological and mechanical correlations on the reliability of vegetated slopes," ASCE-ASME Journal of Risk and Uncertainty in Engineering Systems, Part A: Civil Engineering, vol. 3, no. 4, 2017.

[18] R. B. Nelsen, An Introduction to Copulas, Springer, Berlin, Germany, 2006.

[19] J. H. Chen, J. L. Li, X. L. Xu, L. P. Wan, Y. S. Huang, and H. F. Deng, "Algorithm for generation correlative variables and Monte Carlo simulation of slope reliability," Rock and Soil Mechanics, vol. 38, no. 11, pp. 3341-3346, 2017.

[20] X. Z. Wu, "Modelling dependence structures of soil shear strength data with bivariate copulas and applications to geotechnical reliability analysis," Soils and Foundations, vol. 55, no. 5, pp. 1243-1258, 2015.

[21] B. K. Low, "Reliability analysis of rock slopes involving correlated nonnormals," International Journal of Rock Mechanics and Mining Sciences, vol. 44, no. 6, pp. 922-935, 2007.

[22] D. Li, Y. Chen, W. Lu, and C. Zhou, "Stochastic response surface method for reliability analysis of rock slopes involving correlated non-normal variables," Computers and Geotechnics, vol. 38, no. 1, pp. 58-68, 2011.

[23] J. Ji, C. S. Zhang, Y. Gui, Q. Lü, and J. Kodikara, "New observations on the application of LS-SVM in slope system reliability analysis," Journal of Computing in Civil Engineering, vol. 31, no. 2, pp. 1-9, 2016.

[24] X. P. Zhou, B. Z. Zhu, and L. N. Y. Wong, "A stability analysis of landslides based on random fields, part II: base circle slope," Bulletin of Engineering Geology and the Environment, vol. 78, no. 1, pp. 117-130, 2019.

[25] J. J. Brejda, T. B. Moorman, J. L. Smith, D. L. Karlen, D. L. Allan, and T. H. Dao, "Distribution and variability of surface soil properties at a regional scale," Soil Science Society of America Journal, vol. 64, no. 3, p. 974, 2000. 
[26] G. A. Fenton and D. V. Griffiths, "Bearing-capacity prediction of spatially random $c$, $\phi$ soils," Canadian Geotechnical Journal, vol. 40, no. 1, pp. 54-65, 2003.

[27] S.-H. Jiang, J. Huang, C. Yao, and J. Yang, "Quantitative risk assessment of slope failure in 2-D spatially variable soils by limit equilibrium method," Applied Mathematical Modelling, vol. 47, pp. 710-725, 2017.

[28] K. Harrop-Williams, "Probability distribution of strength parameters in uniform soils," Journal of Engineering $\mathrm{Me}$ chanics, vol. 112, no. 3, pp. 345-350, 1986.

[29] M. E. Harr, Reliability Based Design in Civil Engineering, McGraw-Hill, New York, NY, USA, 1987.

[30] W. S. Forrest and T. L. L. Orr, "Reliability of shallow foundations designed to eurocode 7," Georisk: Assessment and Management of Risk for Engineered Systems and Geohazards, vol. 4, no. 4, pp. 186-207, 2010.

[31] F. Miao, Y. Wu, Y. Xie, F. Yu, and L. Peng, "Research on progressive failure process of Baishuihe landslide based on monte carlo model," Stochastic Environmental Research and Risk Assessment, vol. 31, no. 7, pp. 1683-1696, 2017.

[32] W. Qin, X. Li, and J. Chen, "Study of two dimensional index system of slope stability based on gumbel extreme value I distribution," Journal of China Three Gorges University (Natural Sciences), vol. 39, no. 5, pp. 12-16, 2017.

[33] N. Khalili and M. H. Kabbaz, "A unique relationship for $\chi$ for the determination of the shear strength of unsaturated soils," Géotechnique, vol. 51, no. 5, pp. 477-478, 2001.

[34] Y. Wu, X. Zhou, Y. Gao, L. Zhang, and J. Yang, "Effect of soil variability on bearing capacity accounting for non-stationary characteristics of undrained shear strength," Computers and Geotechnics, vol. 110, pp. 199-210, 2019.

[35] Y. Wu, R. Li, Y. Gao, N. Zhang, and F. Zhang, "Simple and efficient method to simulate homogenous multidimensional Non-Gaussian vector fields by the spectral representation method," Journal of Engineering Mechanics, vol. 143, no. 12, article 06017016, 2017.

[36] C. Huang and D. Ruan, "Fuzzy risks and an updating algorithm with new observations," Risk Analysis, vol. 28, no. 3, pp. 681-694, 2008.

[37] F. Q. Gong, S. Q. Hou, and X. Yan, "Probability model deduction method of Mohr-Coulomb criteria parameters based on normal information diffusion principle," Chinese Journal of Rock Mechanics and Engineering, vol. 32, no. 11, pp. 2225-2234, 2013.

[38] T. L. Huang, F. Q. Gong, and T. Y. Wu, "Orthogonal polynomial inference method of the probability distribution function for small samples of geotechnical parameters," Engineering Mechanics, vol. 34, no. 8, pp. 85-95, 2017.

[39] L. He, Z. Lu, and X. Li, "Failure-mode importance measures in structural system with multiple failure modes and its estimation using copula," Reliability Engineering and System Safety, vol. 174, pp. 53-59, 2018.

[40] X.-S. Tang, D.-Q. Li, C.-B. Zhou, and K.-K. Phoon, “Copulabased approaches for evaluating slope reliability under incomplete probability information," Structural Safety, vol. 52, pp. 90-99, 2015.

[41] M. R. Melchiori, "Which archimedean copula is the right one?," Social Science Research Network Electronic Journal, pp. 1-19, 2000.

[42] H. Akaike, "A new look at the statistical model identification," IEEE Transactions on Automatic Control, vol. 19, no. 6, pp. 716-723, 1974.

[43] L. Hao, L.-Z. Yang, and J.-M. Gao, "The application of information diffusion technique in probabilistic analysis to grassland biological disasters risk," Ecological Modelling, vol. 272, pp. 264-270, 2014.

[44] X. Z. Wang, "The theory, method and robustness of the parameter estimation based on the principle of information spread," Journal of Wuhan Technical University of Surveying and Mapping, vol. 24, no. 3, pp. 240-244, 1999.

[45] N. Smirnov, "Table for estimating the goodness of fit of empirical distributions," The Annals of Mathematical Statistics, vol. 19, no. 2, pp. 279-281, 1948.

[46] H. B. Li, J. Zhao, and T. J. Li, "Triaxial compression tests on a granite at different strain rates and confining pressures," International Journal of Rock Mechanics and Mining Sciences, vol. 36, no. 8, pp. 1057-1063, 1999. 


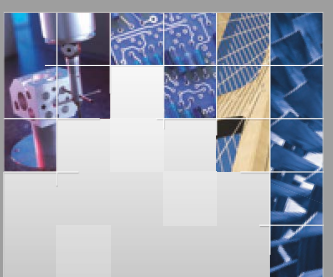

\section{Enfincering}
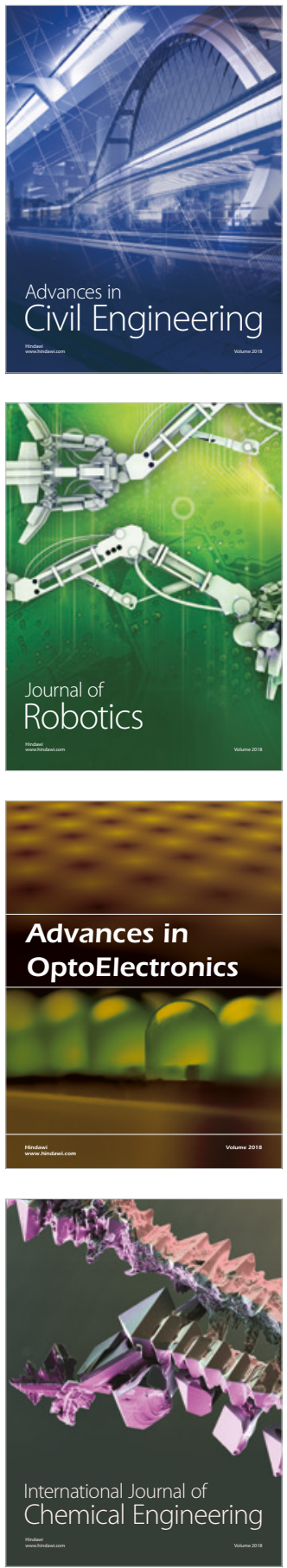

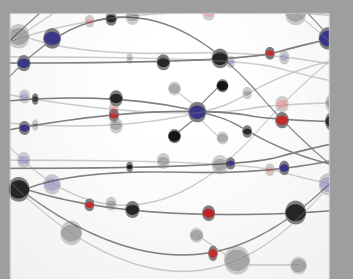

\section{Rotating \\ Machinery}

The Scientific World Journal

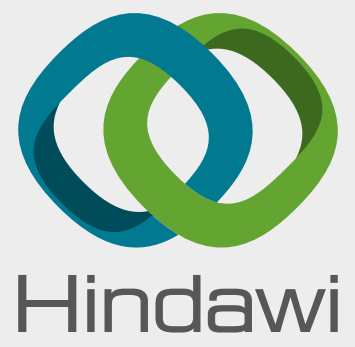

Submit your manuscripts at

www.hindawi.com
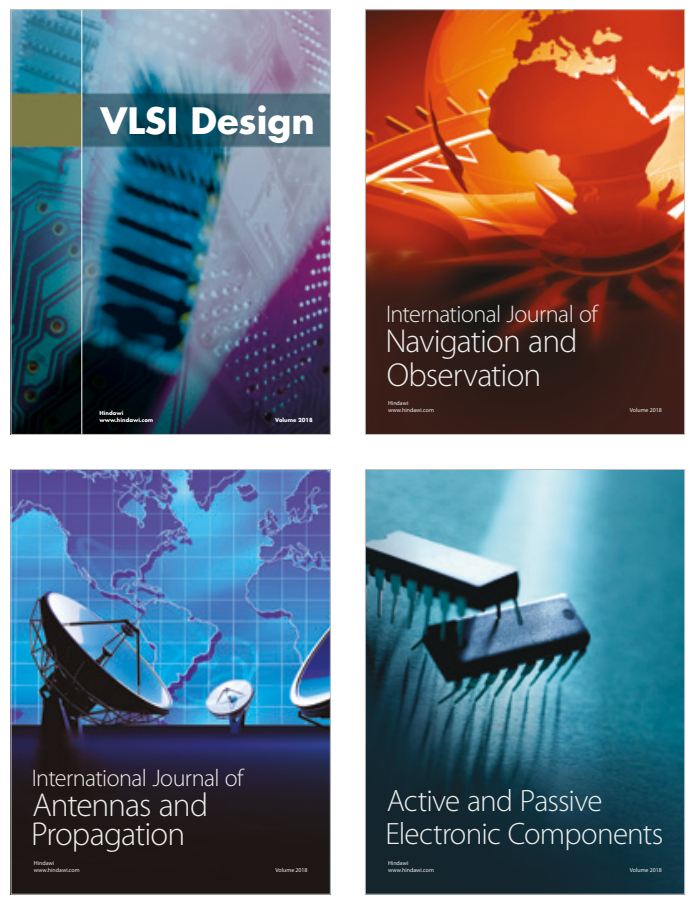
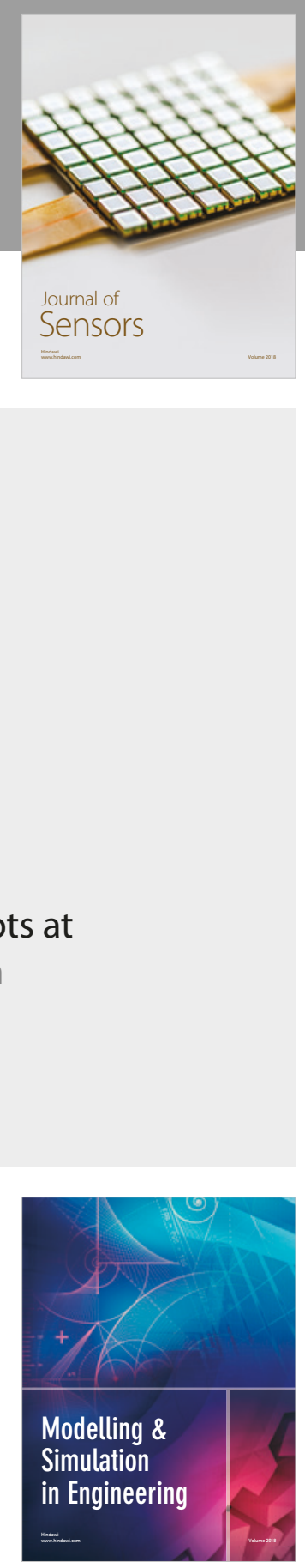

\section{Advances \\ Multimedia}
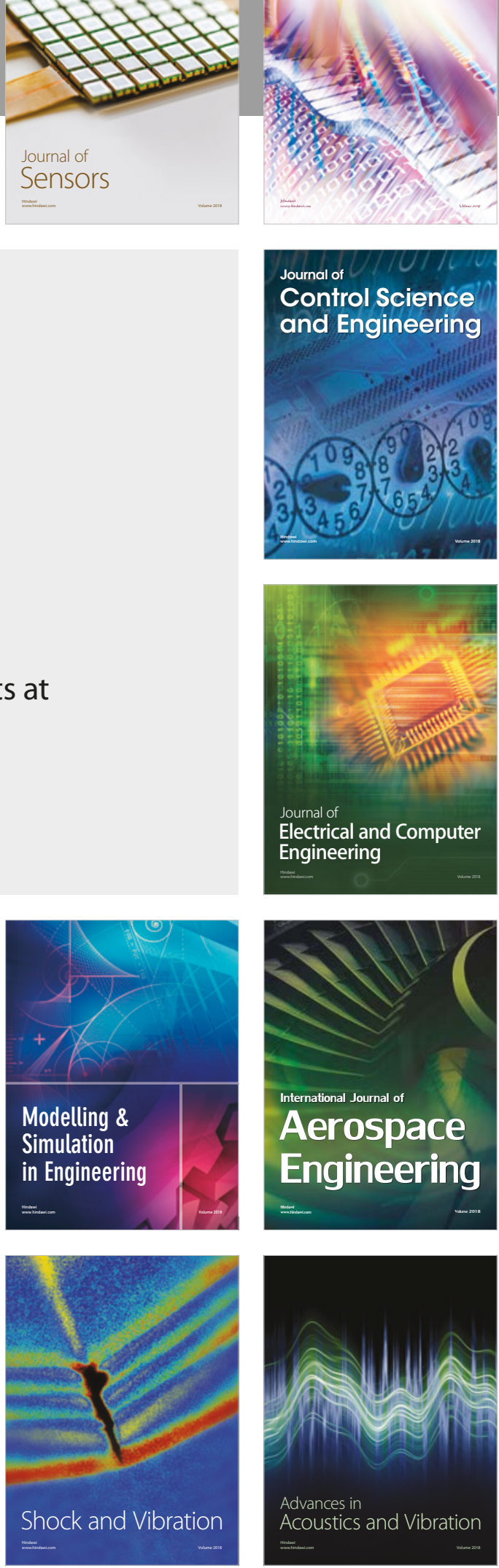